\title{
Impulsive Transfers Between Lissajous Libration Point Orbits
}

\author{
Elisabet Canalias ${ }^{(1)}$, Jordi Cobos $^{(2)}$ and Josep J. Masdemont ${ }^{(1) *}$
}

July 16, 2003

(1) IEEC \& Departament de Matemàtica Aplicada I, Universitat Politècnica de Catalunya, Diagonal 647, 08028 Barcelona, Spain. josep@barquins.upc.es.

(2) TERMA, Flight Dynamics Division, ESA/ESOC, Robert-Bosch-Str. 5, 64293 Darmstadt, Germany.

\begin{abstract}
A complete methodology for the transfer between two Lissajous orbits around the same collinear equilibrium point is developed making use of the geometry of the phase space around these points. The result of the study gives both strategies to change the amplitudes of the Lissajous orbit and simple procedures to avoid the exclusion zone in an optimal way. The presentation is centered on the linear problem which provides good initial guesses for orbits of small amplitude, like the requirements for the mission FIRST/Planck of the European Space Agency (ESA), where part of the results of this paper were used for the mission design.
\end{abstract}

Keywords: Restricted three body problem, Lissajous orbits, invariant manifolds, transfer orbits, exclusion zone.

${ }^{*}$ Corresponding author 


\section{Contents}

1 Introduction 3

2 The Methodology 3

2.1 Changing the in-plane amplitude $\ldots \ldots \ldots \ldots \ldots \ldots \ldots \ldots \ldots \ldots \ldots \ldots \ldots \ldots \ldots$

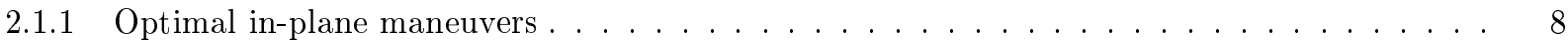

2.1 .2 Changing the in-plane phase $\ldots \ldots \ldots \ldots \ldots \ldots \ldots \ldots$

2.2 Out-of-Plane maneuvers $\ldots \ldots \ldots \ldots \ldots \ldots \ldots \ldots \ldots$

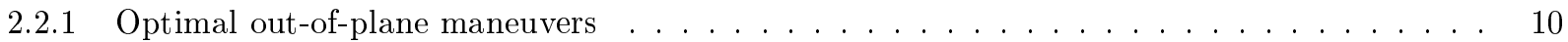

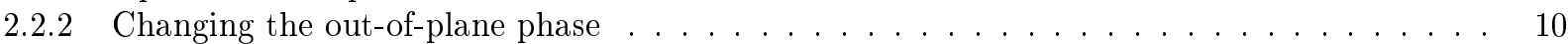

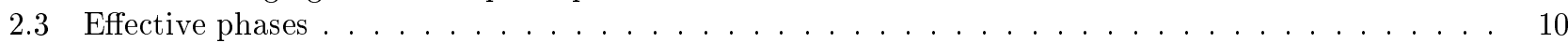

3 Eclipse Avoidance and LOEWE Strategies 11

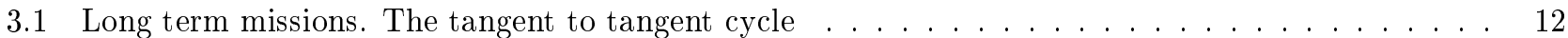

3.1.1 Summary of results for the tangent to tangent cycle $\ldots \ldots \ldots \ldots \ldots$

3.1 .2 Some comments on non-square Lissajous . . . . . . . . . . . . . . . . . . . . 15

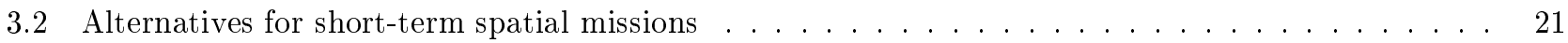

4 Changing the Amplitudes $\quad 22$

4.0.1 Increasing the size of a square Lissajous with a combined maneuver . . . . . . . . . . . 24

4.0.2 Decreasing the size of a square Lissajous with a combined maneuver . . . . . . . . . . 25

4.0.3 Eclipse avoidance in combined maneuvers to change the amplitude . . . . . . . . . . 27

$\begin{array}{llr}5 & \text { Conclusions } & 27\end{array}$

$\begin{array}{lr}\text { References } & 29\end{array}$ 


\section{Introduction}

Libration orbits in a vicinity of the collinear equilibrium points of the Earth-Sun system, $\mathrm{L}_{1}$ about $1.5 \times 10^{6} \mathrm{~km}$ from the Earth towards the Sun and $\mathrm{L}_{2}$ at about the same distance away from the Sun, have been proved to be suitable locations for space missions from 1978 when ISEE-3 was the first spacecraft launched to a halo orbit around $\mathrm{L}_{1}$ (see [5]) till the SOHO spacecraft launched in 1995 (see [22]) which is still a in a halo orbit around $\mathrm{L}_{1}$.

Since the collinear libration points $\mathrm{L}_{1}$ and $\mathrm{L}_{2}$ provide the requirements of a highly stable thermal environmental and sky viewing conditions unobstructed by Earth and Sun, recently many astronomy missions have been considered to be placed there. Just to cite a few of them, FIRST, Planck and GAIA are missions in the ESA Scientific Program for which a class of orbits near $\mathrm{L}_{2}$ have been selected. Orbits around $\mathrm{L}_{2}$ are also going to be used for future astronomy missions as the Next Generation Space Telescope (NGST). Again in the NASA Scientific Program missions like GENESIS, TPF, or even locations for space stations have been considered ([6],[21], [15]).

Instability is a basic dynamical property of the collinear libration points (see [23]). This property is inherited by all the orbits which exist around the libration points (see [7], [9] and [11] for the description of the neighborhood of the libration point orbits). A spacecraft placed in a libration point orbit, will move away from it because of the small perturbations of the orbit that cannot be avoided.

Moreover to place a spacecraft in the equilibrium point is not feasible for two reasons. The fist one is that the amount of propellant, $\Delta v$, to stop the spacecraft at the libration point would be very large, and the second one is that for the $\mathrm{L}_{1}$ case, the equilibrium point appears as a spot in the solar disc. The radiation coming from the sun would make the communication with the satellite impossible. Therefore, an exclusion zone around the solar disc, when the satellite is seen from the Earth, has to be avoided. In a similar way, and exclusion zone around $\mathrm{L}_{2}$ has to be avoided to skip the Earth half-shadow as a requirement of the mission.

Traditionally, the exclusion zone has been avoided by selecting the so-called halo orbits. Halo orbits are a family of periodic orbits which have the equilibrium point inside the bounded component when they are observed from the Earth. The main disadvantage of halo orbits is that they appear very elongated with a big excursion in the direction of the ecliptic plane with respect to the small excursion of the out-of-plane component. This fact makes the communication with the satellite difficult, since it has to be kept pointing towards the Earth at the end of the big excursions. Some strategies to transfer between halo orbits, which are aimed at similar objectives as this paper, can be found in [7] and [8].

There is also a family of orbits around the libration points which are well suited for space missions. They are the so-called Lissajous orbits (see [16] and [11] for an exhaustive description). Lissajous orbits appear as quasi-periodic orbits filling any chosen rectangle around the libration point as seen from the Earth. Its drawback is that at certain times they cross the exclusion zone and so, maneuvers are required to avoid crossings. Studies to avoid the exclusion zones using $z$-maneuvers were first considered in [19] and developed in [3], [10] and [4] for the Earth-Moon case. More recent results concerning the Sun-Earth case can be found in [17] and [7].

The strategy described in this paper gives a new way of both transferring between Lissajous orbits and avoiding the exclusion zone with little cost. As a result, the former $z$-maneuver strategies also appear as a particular case of the methodology. The development of this methodology started during the mission analysis of FIRST, Planck and GAIA of the European Space Agency and actually it is going to be applied for missions FIRST and Planck.

FIRST is the cornerstone project in the ESA Science Program dedicated to far infrared Astronomy. Planck, renamed from COBRAS/SAMBA, is to map the microwave background over the whole sky and is now combined with FIRST for a launch in 2007. Several possible options where considered during the orbit analysis work. The final one adopted was the so-called "Carrier". Both spacecraft will be launched by the same Ariadne 5, but will separate after launch. For this option, the optimum solution is a free transfer to a large amplitude Lissajous orbit. FIRST will remain in this orbit whereas Planck, of much less mass, will perform a size reduction maneuver.

\section{The Methodology}

Let us describe the classical Restricted Three Body Problem (RTBP). Consider the motion of an infinitesimal particle (spacecraft) under the gravitational attraction of the two point like masses called primaries (Sun and Earth). The attraction of the infinitesimal particle on the primaries is neglected so the primaries are describing Keplerian orbits around their common center of mass. The study of the motion of the infinitesimal particle is known as RTBP. Here we will assume that the primaries are moving on a circular orbit. 


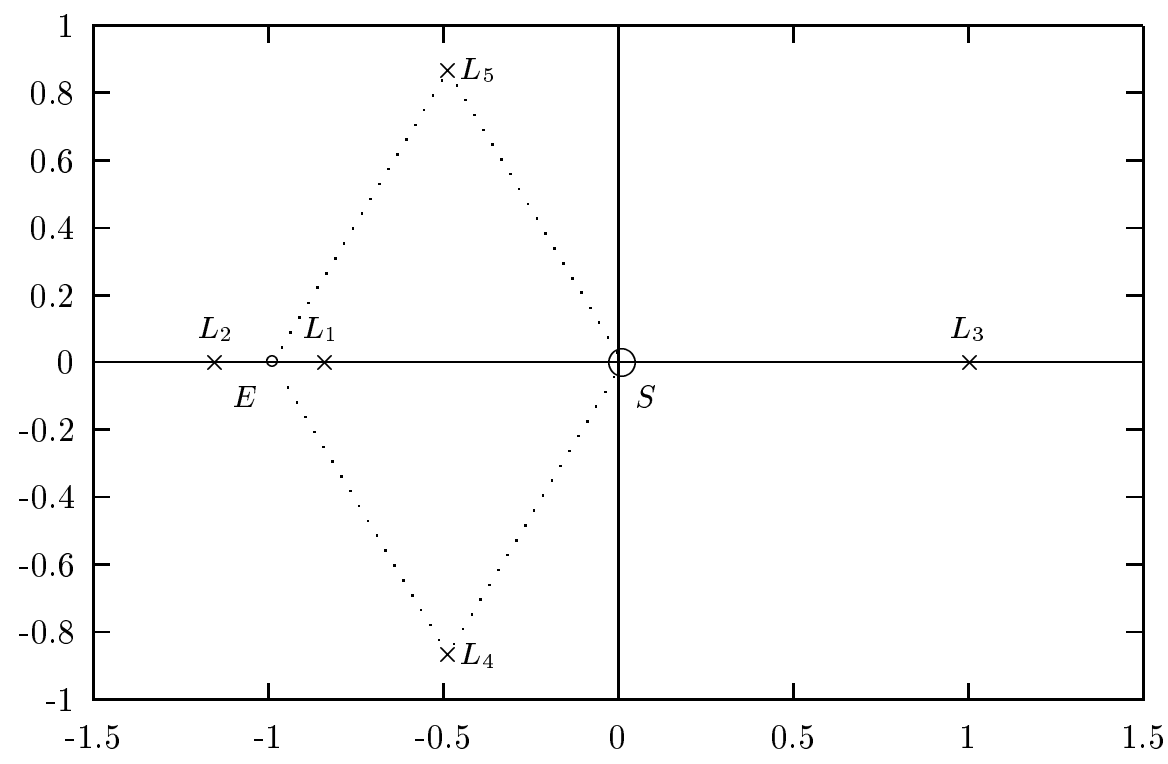

Figure 1: The five equilibrium points of the RTBP.

To simplify the equations of motion, let us take units of mass, length and time such that the sum of the masses of the primaries, the gravitational constant and the period of the primaries is 1,1 and $2 \pi$ respectively. With these units the distance between the primaries is also 1 . We denote by $\mu$ the mass of the smallest primary $\left(\mu=3.040423398444176 \times 10^{-6}\right.$ for the Sun-Earth+Moon system according to JPL ephemeris DE403).

We use the (non-inertial) synodic coordinate system, centered at the center of mass of the primaries, and rotating in such a way that they are at fixed positions on the $X$ axis. The orientation of the $X$ axis is given by the line that goes from the smallest to the biggest primary. The $Z$ axis has the direction given by the angular motion of the primaries and the $Y$ axis is chosen orthogonal to the previous ones to have a positively oriented coordinate system. In this reference frame the small primary of mass $\mu$ is located at $(\mu-1,0,0)$ and the primary of mass $1-\mu$ at $(\mu, 0,0)$. The differential equations of motion in this reference frame are (see [23]),

$$
\ddot{X}-2 \dot{Y}=\frac{\partial \Omega}{\partial X}, \quad \ddot{Y}+2 \dot{X}=\frac{\partial \Omega}{\partial Y}, \quad \ddot{Z}=\frac{\partial \Omega}{\partial Z},
$$

where, $\Omega(X, Y, Z)=\frac{1}{2}\left(X^{2}+Y^{2}\right)+\frac{1-\mu}{r_{1}}+\frac{\mu}{r_{2}}+\frac{1}{2} \mu(1-\mu)$, and $r_{1}, r_{2}$ denote the distances from the spacecraft to the primaries. $r_{1}^{2}=(X-\mu)^{2}+Y^{2}+Z^{2}$, and $r_{2}^{2}=(X+1-\mu)^{2}+Y^{2}+Z^{2}$.

It is well known that the RTBP has five equilibrium points. Three of them are on the $\mathrm{X}$ axis separated by the two primaries. They are called the collinear libration points $\mathrm{L}_{1}, \mathrm{~L}_{2}$ and $\mathrm{L}_{3}$. The remaining two, known as $\mathrm{L}_{4}$ and $\mathrm{L}_{5}$, are also in the plane $Z=0$ forming an equilateral triangle with the primaries, (see Fig. 1)

Following [20], let us write the equations of motion (1) centered in a selected collinear libration point and at the same time scaling the longitude in such a way that the new distance from the origin (equilibrium point) to the closest primary be equal to one.

The distance from $\mathrm{L}_{j}$ to the closest primary is given by the Euler quintic equation (see [23]),

$$
\begin{array}{r}
\gamma^{5} \mp(3-\mu) \gamma^{4}+(3-2 \mu) \gamma^{3}-\mu \gamma^{2} \pm 2 \mu \gamma-\mu=0, \quad j=1,2 \\
\gamma^{5}+(2+\mu) \gamma^{4}+(1+2 \mu) \gamma^{3}-(1-\mu) \gamma^{2}-2(1-\mu) \gamma-(1-\mu)=0, \quad j=3
\end{array}
$$

Once the equilibrium point is selected, and the corresponding value of $\gamma$ computed, the change of coordinates we choose is given by,

$$
X=\mp \gamma x+\mu+a, \quad Y=\mp \gamma y, \quad Z=\gamma z
$$

where the upper sign corresponds to $\mathrm{L}_{1,2}$, the lower one to $\mathrm{L}_{3}, a=-1+\gamma$ for $\mathrm{L}_{1}, a=-1-\gamma$ for $\mathrm{L}_{2}$, and $a=\gamma$ for $\mathrm{L}_{3}$. 
In this new reference system, the form of the linearized equations is,

$$
\left.\begin{array}{rl}
\ddot{x}-2 \dot{y}-\left(1+2 c_{2}\right) & x=0 \\
\ddot{y}+2 \dot{x}+\left(c_{2}-1\right) y & =0 \\
\ddot{z}+c_{2} z & =0
\end{array}\right\}
$$

where, with the same notation as [9], $c_{2}$ is a constant depending only on the masses:

$$
c_{2}=\frac{1}{\gamma^{3}}\left(\mu+(1-\mu) \frac{\gamma^{3}}{(1 \mp \gamma)^{3}}\right) \quad \text { for } \mathrm{L}_{1}, \mathrm{~L}_{2}, \quad c_{2}=\frac{1}{\gamma^{3}}\left(1-\mu+\mu \frac{\gamma^{3}}{(1+\gamma)^{3}}\right) \quad \text { for } \mathrm{L}_{3} \text {. }
$$

Quasi-periodic solutions of the linear system (3) are characterized by an harmonic motion in the ecliptic $x y$ plane (also known as "in-plane component") and an uncoupled oscillation in the $z$ direction (also known as "out-ofplane component") with a different period. The general solution is obtained by adding the hyperbolic exponential parts, which due to the Hamiltonian character of the RTBP equations have both stable and unstable components with opposite exponents,

$$
\left.\begin{array}{l}
x(t)=A_{1} e^{\lambda t}+A_{2} e^{-\lambda t}+A_{3} \cos \omega t+A_{4} \sin \omega t \\
y(t)=c A_{1} e^{\lambda t}-c A_{2} e^{-\lambda t}-\bar{k} A_{4} \cos \omega t+\bar{k} A_{3} \sin \omega t \\
z(t)=A_{5} \cos \nu t+A_{6} \sin \nu t
\end{array}\right\}
$$

where $A_{i}$ are arbitrary constants and $c, \bar{k}, \omega, \lambda$ and $\nu$ are constants depending on $c_{2}$ only:

$\omega=\sqrt{\frac{2-c_{2}+\sqrt{9 c_{2}^{2}-8 c_{2}}}{2}}, \quad \nu=\sqrt{c_{2}}, \quad c=\frac{\lambda^{2}-1-2 c_{2}}{2 \lambda}, \quad \lambda=\sqrt{\frac{c_{2}-2+\sqrt{9 c_{2}^{2}-8 c_{2}}}{2}}, \quad \bar{k}=\frac{-\left(\omega^{2}+1+2 c_{2}\right)}{2 \omega}$.

Since the in-plane and out-of-plane motions are decoupled, we get the following relations between trajectories, $(x(t), y(t), z(t), \dot{x}(t), \dot{y}(t), \dot{z}(t))$ and the first integrals (constants of motion) $A_{1}, A_{2}, \ldots, A_{6}$,

$$
\begin{aligned}
\left(\begin{array}{l}
x \\
y \\
\dot{x} \\
\dot{y}
\end{array}\right)= & \left(\begin{array}{cccc}
e^{\lambda t} & e^{-\lambda t} & \cos \omega t & \sin \omega t \\
c e^{\lambda t} & -c e^{-\lambda t} & \bar{k} \sin \omega t & -\bar{k} \cos \omega t \\
\lambda e^{\lambda t} & -\lambda e^{-\lambda t} & -\omega \sin \omega t & \omega \cos \omega t \\
c \lambda e^{\lambda t} & c \lambda e^{-\lambda t} & \bar{k} \omega \cos \omega t & \bar{k} \omega \sin \omega t
\end{array}\right)\left(\begin{array}{c}
A_{1} \\
A_{2} \\
A_{3} \\
A_{4}
\end{array}\right) \\
& \left(\begin{array}{c}
z \\
\dot{z}
\end{array}\right)=\left(\begin{array}{cc}
\cos \nu t & \sin \nu t \\
-\nu \sin \nu t & \nu \cos \nu t
\end{array}\right)\left(\begin{array}{c}
A_{5} \\
A_{6}
\end{array}\right)
\end{aligned}
$$

Inverting the system we get the first integrals of (4) in terms of a given state vector at time $t,(x(t), y(t), z(t)$, $\dot{x}(t), \dot{y}(t), \dot{z}(t))$ :

$$
\begin{aligned}
\left(\begin{array}{c}
A_{1} \\
A_{2} \\
A_{3} \\
A_{4}
\end{array}\right)= & \left(\begin{array}{cccc}
\frac{-\bar{k} \omega}{2 d_{1}} e^{-\lambda t} & \frac{\omega}{2 d_{2}} e^{-\lambda t} & \frac{\bar{k}}{2 d_{2}} e^{-\lambda t} & \frac{1}{2 d_{1}} e^{-\lambda t} \\
\frac{-k \omega}{2 d_{1}} e^{\lambda t} & \frac{-\omega}{2 d_{2}} e^{\lambda t} & \frac{-k}{2 d_{2}} e^{\lambda t} & \frac{1}{2 d_{1}} e^{\lambda t} \\
\frac{c \lambda}{d_{1}} \cos \omega t & \frac{\lambda}{d_{2}} \sin \omega t & \frac{-c}{d_{2}} \sin \omega t & \frac{-1}{d_{1}} \cos \omega t \\
\frac{c \lambda}{d_{1}} \sin \omega t & \frac{-\lambda}{d_{2}} \cos \omega t & \frac{c}{d_{2}} \cos \omega t & -\frac{1}{d_{1}} \sin \omega t
\end{array}\right)\left(\begin{array}{l}
x \\
y \\
\dot{x} \\
\dot{y}
\end{array}\right) \\
& \left(\begin{array}{c}
A_{5} \\
A_{6}
\end{array}\right)=\left(\begin{array}{cc}
\cos \nu t & \frac{-1}{\nu} \sin \nu t \\
-\sin \nu t & \frac{1}{\nu} \cos \nu t
\end{array}\right)\left(\begin{array}{c}
z \\
\dot{z}
\end{array}\right)
\end{aligned}
$$

where $d_{1}=c \lambda-\bar{k} \omega$ and $d_{2}=c \omega+\bar{k} \lambda$.

Finally, it is also convenient to look at the oscillatory solution of the linear part as having an amplitude and a phase,

$$
\left.\begin{array}{l}
x(t)=A_{1} e^{\lambda t}+A_{2} e^{-\lambda t}+A_{x} \cos (\omega t+\phi) \\
y(t)=c A_{1} e^{\lambda t}-c A_{2} e^{-\lambda t}+\bar{k} A_{x} \sin (\omega t+\phi) \\
z(t)=A_{z} \cos (\nu t+\psi)
\end{array}\right\}
$$

where the relations are $A_{3}=A_{x} \cos \phi, A_{4}=-A_{x} \sin \phi, A_{5}=A_{z} \cos \psi$ and $A_{6}=-A_{z} \sin \psi$. 
We note that choosing, $A_{1}=A_{2}=0$, we obtain a periodic motion in the $x y$ components together with a periodic motion in $z$ of a different period. This represents the Lissajous orbits in the linearized restricted circular three-body problem, $A_{x}, A_{z}$ being the maximum in-plane and out-of-plane amplitudes respectively. The first integrals $A_{1}$ and $A_{2}$ are directly related to the unstable and stable manifold of the linear Lissajous orbit. For instance, the relation $A_{1}=0, A_{2} \neq 0$, defines a stable manifold. Any orbit orbit verifying this condition will tend forward in time to the Lissajous (or periodic) orbit defined by $A_{x}$ and $A_{z}$, since the term containing the $A_{2}$-component in (4) will die out. A similar fact happens when $A_{1} \neq 0, A_{2}=0$, but now backwards in time. Then, this later condition defines an unstable manifold.

In our analysis of the transfer we want to avoid unstable motions forward in time and this means that we require the condition $A_{1}=0$. Using (6) this is equivalent to,

$$
\frac{\bar{k}}{d_{2}} \dot{x}+\frac{1}{d_{1}} \dot{y}=\frac{\bar{k} \omega}{d_{1}} x-\frac{\omega}{d_{2}} y
$$

It is important to note that, given a position $x, y$ of the spacecraft, we have an explicit formula for the set of possible velocities, $\dot{x}, \dot{y}$, for which escape is avoided.

Let us assume now that we are on a trajectory verifying the non escape condition $A_{1}=0$ (for instance on a Lissajous orbit). If we want to keep the non escape condition after performing a maneuver $(\Delta \dot{x}, \Delta \dot{y})$, because of the linearity of the equations, it is mandatory that,

$$
\frac{\bar{k}}{d_{2}} \Delta \dot{x}+\frac{1}{d_{1}} \Delta \dot{y}=0
$$

and so the corollary is that the maneuvers preserving $A_{1}=0$ are orthogonal to the vector $\left(\frac{\bar{k}}{d_{2}}, \frac{1}{d_{1}}\right)$, this is, $(\Delta \dot{x}, \Delta \dot{y})=a\left(\frac{1}{d_{1}}, \frac{-\bar{k}}{d_{2}}\right)$ where $a \in \mathbb{R}$. This expression will be used in its equivalent form,

$$
(\Delta \dot{x}, \Delta \dot{y})=\frac{\alpha}{\sqrt{c^{2}+\bar{k}^{2}}}\left(d_{2},-\bar{k} d_{1}\right), \quad \alpha \in \mathbb{R}
$$

since now, $|\alpha|$, indicates the size of the maneuver.

Due to the decoupling between the in-plane and out-of-plane motions in the linear theory, the problem of finding a minimum $\Delta v$ transfer between two Lissajous orbits of different size can also, in a first step, be decoupled in two problems. So let us discuss the in-plane amplitude reduction, and with a similar approach, the out-of-plane amplitude reduction. Finally, in section 4.0.3 we will discuss in which cases these two maneuvers may combine by vector addition into a single one which possibly has a smaller modulus because of the parallelogram rule for vector addition. This will give us criteria on how to distribute the overall size reduction of the Lissajous orbit between the amplitude reductions in the $x y$ and $z$ components in an optimum way.

\subsection{Changing the in-plane amplitude}

Let us assume that at a given time $t_{m}$ we perform a maneuver $(\Delta \dot{x}, \Delta \dot{y})$. The central part (i.e. the libration terms) will change from an initial in-plane amplitude $A_{x}^{(i)}$ to a final one $A_{x}^{(f)}$ given by,

$$
\begin{aligned}
A_{x}^{(f)}{ }^{2}= & A_{3}^{(f)}{ }^{2}+A_{4}^{(f)}{ }^{2}=\left(\frac{c \lambda}{d_{1}} x\left(t_{m}\right) \cos \omega t_{m}+\frac{\lambda}{d_{2}} y\left(t_{m}\right) \sin \omega t_{m}-\right. \\
& \left.-\frac{c}{d_{2}}\left(\dot{x}\left(t_{m}^{-}\right)+\Delta \dot{x}\right) \sin \omega t_{m}-\frac{1}{d_{1}}\left(\dot{y}\left(t_{m}^{-}\right)+\Delta \dot{y}\right) \cos \omega t_{m}\right)^{2}+\left(\frac{c \lambda}{d_{1}} x\left(t_{m}\right) \sin \omega t_{m}-\right. \\
& \left.-\frac{\lambda}{d_{2}} y\left(t_{m}\right) \cos \omega t_{m}+\frac{c}{d_{2}}\left(\dot{x}\left(t_{m}^{-}\right)+\Delta \dot{x}\right) \cos \omega t_{m}-\frac{1}{d_{1}}\left(\dot{y}\left(t_{m}^{-}\right)+\Delta \dot{y}\right) \sin \omega t_{m}\right)^{2} .
\end{aligned}
$$

where $\dot{x}\left(t_{m}^{-}\right)$has to be understood as $\lim _{t \rightarrow t_{m}, t<t m} \dot{x}(t)$, and analogously for $\dot{y}\left(t_{m}^{-}\right)$. These are the $x$ and $y$ velocities just before performing the maneuver. 
After some cumbersome algebra which introduces the expressions of the amplitudes $A_{3}^{(i)}$ and $A_{4}^{(i)}$ in terms of $x\left(t_{m}\right), y\left(t_{m}\right), \dot{x}\left(t_{m}^{-}\right)$and $\dot{y}\left(t_{m}^{-}\right)$we obtain,

$$
A_{x}^{(f)}=A_{x}^{(i)}{ }^{2}+\frac{c^{2}}{d_{2}^{2}}(\Delta \dot{x})^{2}+\frac{1}{d_{1}^{2}}(\Delta \dot{y})^{2}-2 \frac{c \lambda}{d_{2}^{2}} y\left(t_{m}\right) \Delta \dot{x}+2 \frac{c^{2}}{d_{2}^{2}} \dot{x}\left(t_{m}^{-}\right) \Delta \dot{x}-2 \frac{c \lambda}{d_{1}^{2}} x\left(t_{m}\right) \Delta \dot{y}+2 \frac{1}{d_{1}^{2}} \dot{y}\left(t_{m}^{-}\right) \Delta \dot{y} .
$$

We note that this expression is valid in the most generic case, this is, for any maneuver $(\Delta \dot{x}, \Delta \dot{y})$. Let us assume now that the maneuver is done when the satellite is on a Lissajous orbit. In this case for $t<t_{m}$ we have,

$$
\begin{array}{ll}
x(t)=A_{x}^{(i)} \cos (\omega t+\phi) & y(t)=\bar{k} A_{x}^{(i)} \sin (\omega t+\phi) \\
\dot{x}(t)=-A_{x}^{(i)} \omega \sin (\omega t+\phi) & \dot{y}(t)=\bar{k} A_{x}^{(i)} \omega \cos (\omega t+\phi)
\end{array}
$$

being the expressions for $x(t)$ and $y(t)$ also valid when $t=t_{m}$.

So until just before the maneuver we have, $\dot{x}=\frac{-\omega}{\bar{k}} y, \dot{y}=\bar{k} \omega x$, moreover, $A_{x}^{(i)}{ }^{2}=x^{2}+\frac{1}{k^{2}} y^{2}$. Using these facts and relations among the constants such as, $\frac{c \omega}{d_{2}}+\frac{\lambda \bar{k}}{d_{2}}=\frac{c \lambda}{d_{1}}-\frac{\bar{k} \omega}{d_{1}}=1$, again after some more algebra we obtain,

$$
A_{x}^{(f)}=\left(\frac{c}{d_{2}} \Delta \dot{x}-\frac{1}{\bar{k}} y\left(t_{m}\right)\right)^{2}+\left(\frac{1}{d_{1}} \Delta \dot{y}-x\left(t_{m}\right)\right)^{2}
$$

which gives us the final in-plane amplitude that is reached when a maneuver $(\Delta \dot{x}, \Delta \dot{y})$ is done at time $t_{m}$ in the $x\left(t_{m}\right), y\left(t_{m}\right)$ position corresponding to a Lissajous orbit. We note that up to this point we have not still required the non-escape condition to the maneuver.

Let us now assume that the maneuver at time $t_{m}$ is done in order to perform a transfer from a Lissajous orbit to another one with a different in-plane amplitude, leaving the out-of-plane motion untouched. The natural approach is to insert the satellite into the stable manifold of the final Lissajous orbit from the departure position when possible. The maneuver will select $A_{x}^{(f)}$ keeping $A_{1}=0$. The new $A_{2}$-term which appear as a consequence of the maneuver tends to vanish, since it is accompanied by the exponential decay in (4). The result is that we will reach the final orbit asymptotically with no more maneuvers.

For this strategy we have only to select the maneuver $(\Delta \dot{x}, \Delta \dot{y})$ in the direction of the non escape condition according to formula (9). Then for the final in-plane amplitude we have,

$$
A_{x}^{(f)}{ }^{2}=\alpha^{2}+\frac{2 \alpha}{\sqrt{c^{2}+\bar{k}^{2}}}\left(\frac{-c}{\bar{k}} y\left(t_{m}\right)+\bar{k} x\left(t_{m}\right)\right)+A_{x}^{(i)}{ }^{2}
$$

where again, $A_{x}^{(i)}{ }^{2}=\frac{1}{k^{2}} y^{2}+x^{2}$, has been used.

At this point it is convenient to introduce a constant angle, $\beta$, defined as the slope of the vector $(c, \bar{k})$. This is, $(\cos \beta, \sin \beta)=\left(\frac{c}{\sqrt{c^{2}+\bar{k}^{2}}}, \frac{\bar{k}}{\sqrt{c^{2}+\bar{k}^{2}}}\right)$. Then, defining the function,

$$
p(t)=A_{x}^{(i)} \sin \left(\omega t+\phi_{i}-\beta\right)
$$

the expression for the final amplitude $A_{x}^{(f)}$ becomes,

$$
A_{x}^{(f)}=\alpha^{2}-2 p\left(t_{m}\right) \alpha+A_{x}^{(i)}
$$

This means that, for a given time, the magnitude of the maneuver necessary to reach a target in-plane amplitude is given by the quadratic equation, $\alpha^{2}-2 p\left(t_{m}\right) \alpha-\left({A_{x}^{(f)}}^{2}-{A_{x}^{(i)}}^{2}\right)^{2}$, and so, $\alpha=p\left(t_{m}\right) \pm \sqrt{p^{2}\left(t_{m}\right)+\left(A_{x}^{(f)}{ }^{2}-{A_{x}^{(i)}}^{2}\right)}$. Moreover, using the definition of $p(t)$, we have,

$$
\alpha=A_{x}^{(i)} \sin \left(\omega t_{m}+\phi_{i}-\beta\right) \pm \sqrt{{A_{x}^{(f)}}^{2}-A_{x}^{(i)}{ }^{2} \cos ^{2}\left(\omega t_{m}+\phi_{i}-\beta\right)}
$$

From this last expression, we observe that if,

- $A_{x}^{(f)} \geq A_{x}^{(i)}$, the transfer maneuver is possible at any time. 
- $A_{x}^{(f)}<A_{x}^{(i)}$, the transfer maneuver is possible only when the expression inside the square root is positive.

More precisely, when $\omega t_{m}+\phi_{i}-\beta \in\left[\delta, \frac{\pi}{2}+\delta\right](\bmod \pi)$, where $\delta=\arccos \left(\frac{A_{x}^{(f)}}{A_{x}^{(i)}}\right)$.

Once the target amplitude $A_{x}^{(f)}$ is selected, we note the two basic possibilities that we have when selecting the maneuver.

- Select $t_{m}$ in such a way that the $\Delta v$ expended in changing the amplitude be a minimum. As we said in (9) this corresponds to the minimum of $|\alpha|$.

- Select $t_{m}$ in such a way that you arrive at the target orbit with a selected phase.

let us comment these possibilities and their implications.

\subsubsection{Optimal in-plane maneuvers}

Let us assume that $A_{x}^{(f)} \neq A_{x}^{(i)}$. Looking for the local minima of $\alpha\left(t_{m}\right)$ we get that performing the maneuver when $t_{m}$ verifies,

$$
\omega t_{m}+\phi_{i}=\beta+\frac{\pi}{2}, \quad \text { or } \quad \omega t_{m}+\phi_{i}=\beta+\frac{3 \pi}{2}, \quad(\text { both } \bmod 2 \pi),
$$

this is, when the angle $\omega t_{m}+\phi_{i}$ is orthogonal to $\beta$, or equivalently, when the satellite on a Lissajous orbit crosses the plane $c x+y=0$. At this point the minimum fuel expenditure for the maneuver, $\Delta v=\left|\alpha_{\text {min }}\right|=\left|A_{x}^{(f)}-A_{x}^{(i)}\right|$ is obtained ${ }^{1}$.

\subsubsection{Changing the in-plane phase}

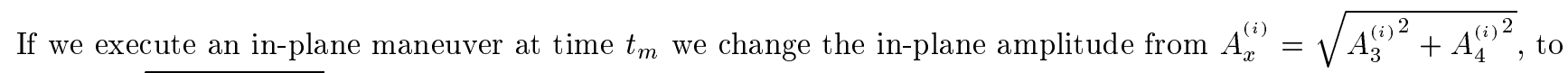
$A_{x}^{(f)}=\sqrt{\left.A_{3}^{(f)}{ }^{2}+A_{4}^{(f)}\right)^{2}}$, which will remain constant along the new trajectory. But depending on $t_{m}$, the values of the components $A_{3}^{(f)}$ and $A_{4}^{(f)}$ will be different, giving as a result the possibility of reaching the target orbit at different phases.

Let us assume that at time $t_{m}$ we perform a maneuver, $(\Delta \dot{x}, \Delta \dot{y})$. Using the equations (6) to compute the first integrals $A_{3}^{(f)}$ and $A_{4}^{(f)}$ in terms of $x\left(t_{m}\right), y\left(t_{m}\right), \dot{x}\left(t_{m}^{+}\right)$and $\dot{y}\left(t_{m}^{+}\right)$, where $\dot{x}\left(t_{m}^{+}\right)=\dot{x}\left(t_{m}^{-}\right)+\Delta \dot{x}$, and analogously for $\dot{y}\left(t_{m}^{+}\right)$(i.e. the velocities just after the maneuver). We obtain,

$$
\begin{aligned}
& A_{3}^{(f)}=A_{3}^{(i)}-\frac{c}{d_{2}} \sin \omega t_{m} \Delta \dot{x}-\frac{1}{d_{1}} \cos \omega t_{m} \Delta \dot{y}, \\
& A_{4}^{(f)}=A_{4}^{(i)}+\frac{c}{d_{2}} \cos \omega t_{m} \Delta \dot{x}-\frac{1}{d_{1}} \sin \omega t_{m} \Delta \dot{y} .
\end{aligned}
$$

Assuming, indeed, that the maneuver $(\Delta \dot{x}, \Delta \dot{y})$ is done in the non escape direction (9) and using the definition of $\beta$, these formulae can be cast into,

$$
A_{3}^{(f)}=A_{3}^{(i)}-\alpha\left(t_{m}\right) \sin \left(\omega t_{m}-\beta\right), \quad A_{4}^{(f)}=A_{4}^{(i)}+\alpha\left(t_{m}\right) \cos \left(\omega t_{m}-\beta\right),
$$

where, if the maneuver is done when the satellite is on a Lissajous orbit, and the target amplitude $A_{x}^{(f)}$ can be effectively reached, the value of $\alpha\left(t_{m}\right)$ is given by (12).

\section{In-plane phase change maneuvers maintaining the amplitude}

As a particular case of changing the phase let us study the case where the in-plane amplitude is maintained. This special case will be very useful for the design of strategies that avoid the exclusion zones.

Let us assume that we perform the maneuver on a Lissajous orbit, $x(t)=A_{x}^{(i)} \cos \left(\omega t+\phi_{i}\right), y(t)=-\bar{k} A_{x}^{(i)} \sin \left(\omega t+\phi_{i}\right)$, with the purpose of arriving asymptotically to another one, $x(t)=A_{x}^{(f)} \cos \left(\omega t+\phi_{f}\right), y(t)=-\bar{k} A_{x}^{(f)} \sin \left(\omega t+\phi_{f}\right)$, with $A_{x}^{(f)}=A_{x}^{(i)}$ that will be just denoted by $A_{x}$ in this subsection. We want to link $\phi_{f}$ with $\phi_{i}$ depending on $t_{m}$.

\footnotetext{
${ }^{1}$ Even in the case $A_{x}^{(f)}<A_{x}^{(i)}$, because in this case the points of discontinuity verify, $\cos \left(\omega t+\phi_{i}-\beta\right)=A_{x}^{(f)} / A_{x}^{(i)}$, giving, $|\alpha|=\sqrt{A_{x}^{(i)^{2}}-A_{x}^{(f)^{2}}}>\left|\alpha_{m i n}\right|$, when $A_{x}^{(f)}<A_{x}^{(i)}$.
} 
In this case, according to (12), the non trivial maneuver at time $t_{m}$ is given by,

$$
\alpha\left(t_{m}\right)=2 A_{x} \sin \left(\omega t_{m}+\phi_{i}-\beta\right)=2 p\left(t_{m}\right) .
$$

Using now (14) and the fact that $A_{3}^{(i)}=A_{x} \cos \phi_{i}, A_{4}^{(i)}=-A_{x} \sin \phi_{i}$, we get the new components for the in-plane amplitude,

$$
\begin{aligned}
& A_{3}^{(f)}=A_{x} \cos \phi_{i}-2 A_{x} \sin \left(\omega t_{m}+\phi_{i}-\beta\right) \sin \left(\omega t_{m}-\beta\right) \\
& A_{4}^{(f)}=-A_{x} \sin \phi_{i}+2 A_{x} \sin \left(\omega t_{m}+\phi_{i}-\beta\right) \cos \left(\omega t_{m}-\beta\right) .
\end{aligned}
$$

And after some trigonometry the computations end up with,

$$
A_{3}^{(f)}=A_{x} \cos \left(2\left(\omega t_{m}-\beta\right)+\phi_{i}\right), \quad A_{4}^{(f)}=A_{x} \sin \left(2\left(\omega t_{m}-\beta\right)+\phi_{i}\right) .
$$

Comparing these expressions with their alternative ones, $A_{3}^{(f)}=A_{x} \cos \phi_{f}$, and $A_{4}^{(f)}=-A_{x}$ sin $\phi_{f}$, we see that the relation between the phases is $\phi_{f}=-2\left(\omega t_{m}-\beta\right)-\phi_{i}$ or equivalently,

$$
\phi_{f}-\phi_{i}=-2\left(\omega t_{m}-\beta+\phi_{i}\right) \quad(\bmod 2 \pi) .
$$

\subsection{Out-of-Plane maneuvers}

Let us assume that a $z$-maneuver is performed at a given time $t_{m}$. Using (7) in a similar way that for the discussion of the in-plane maneuvers, a $\Delta v$ of magnitude $\Delta \dot{z}$ in the $z$ direction changes the first integrals $A_{5}$ and $A_{6}$, according to,

$$
A_{5}^{(f)}=A_{5}^{(i)}-\frac{\Delta \dot{z}}{\nu} \sin \left(\nu t_{m}\right), \quad A_{6}^{(f)}=A_{6}^{(i)}+\frac{\Delta \dot{z}}{\nu} \cos \left(\nu t_{m}\right) .
$$

After little algebra which uses (5) we get the following expression for the $z$-amplitude,

$$
A_{z}^{(f)}{ }^{2}=A_{5}^{(f)}{ }^{2}+A_{6}^{(f)}{ }^{2}=A_{z}^{(i)}{ }^{2}+\frac{2}{\nu^{2}} \dot{z}\left(t_{m}^{-}\right) \Delta \dot{z}+\frac{(\Delta \dot{z})^{2}}{\nu^{2}} .
$$

But according to (8), the motion in the $z$ component till just before the maneuver is, $\dot{z}=-\nu A_{z}^{(i)} \sin \left(\nu t+\psi_{i}\right)$, and so,

$$
A_{z}^{(f)}{ }^{2}=\frac{(\Delta \dot{z})^{2}}{\nu^{2}}-2 \frac{\Delta \dot{z}}{\nu} A_{z}^{(i)} \sin \left(\nu t_{m}+\psi_{i}\right)+A_{z}^{(i)}{ }^{2} .
$$

We note that equations (18) and (19) are completely analogous to equations (14) and (11) of the in-plane case respectively. Essentially, the formal role of $\alpha$ is now played by $\Delta \dot{z} / \nu$ and the former angle, $\beta$, is now zero. Then, the discussion for the out of plane maneuvers follows a parallel way to the one we have done for the in-plane case. We are only going to summarize the results.

The formula for the required $\Delta v$ analogous to (12) is now,

$$
\frac{\Delta \dot{z}}{\nu}=A_{z}^{(i)} \sin \left(\nu t_{m}+\psi_{i}\right) \pm \sqrt{A_{z}^{(f)}{ }^{2}-A_{z}^{(i)} \cos ^{2}\left(\nu t_{m}+\psi_{i}\right)}
$$

and the corresponding discussion is the following. If,

- $A_{z}^{(f)} \geq A_{z}^{(i)}$ the transfer maneuver is possible at any time.

- $A_{z}^{(f)}<A_{z}^{(i)}$ the transfer maneuver is possible only if the expression inside the square root is positive. More precisely, when $\nu t_{m}+\psi_{i} \in\left[\varepsilon, \frac{\pi}{2}+\varepsilon\right](\bmod \pi)$, where $\varepsilon=\arccos \left(\frac{A_{z}^{(f)}}{A_{z}^{(i)}}\right)$. 


\subsubsection{Optimal out-of-plane maneuvers}

When the transfer is possible, the maneuver to change the out-of-plane amplitude from $A_{z}^{(i)}$ to $A_{z}^{(f)}$ is optimal when $t_{m}$ verifies,

$$
\nu t_{m}+\psi_{i}=\frac{\pi}{2}, \quad \text { or } \quad \nu t_{m}+\psi_{i}=\frac{3 \pi}{2}, \quad(\text { both } \bmod 2 \pi) .
$$

In this case, the minimal $\Delta v=|\Delta \dot{z}|$ is given according to, $\Delta \dot{z}=\nu\left(A_{z}^{(i)}-A_{z}^{(f)}\right)$, or $\Delta \dot{z}=\nu\left(A_{z}^{(f)}-A_{z}^{(i)}\right)$, for the $\pi / 2$ or $3 \pi / 2$ cases respectively.

Since the out-of-plane movement is governed by $z(t)=A_{z}^{(i)} \cos \left(\nu t+\psi_{i}\right)$, we note that the optimal times correspond to $z=0$. This is when the satellite crosses the ecliptic plane, which is natural if we think in terms of energy. We remark that the ecliptic plane plays the same role for the out-of-plane maneuvers as the plane $c x+y=0$ plays for the in-plane ones.

\subsubsection{Changing the out-of-plane phase}

Equations (18) give us the components of the final $z$-amplitude $A_{z}^{(f)}$. The corresponding phase, $\psi_{f}$, can be obtained from the definition, $A_{5}^{(f)}=A_{z}^{(f)} \cos \psi_{f}, A_{6}^{(f)}=-A_{z}^{(f)} \sin \psi_{f}$.

In this context we can also consider a maneuver in the $z$-component to change the out-of-plane phase without changing the amplitude. The parallel equation to (15) which gives now the non trivial maneuver is,

$$
\frac{\Delta \dot{z}}{\nu}=2 A_{z} \sin \left(\nu t_{m}+\psi_{i}\right)
$$

and the corresponding change of phase as a function of $t_{m}$ is,

$$
\psi_{f}-\psi_{i}=-2\left(\nu t_{m}+\psi_{i}\right) \quad(\bmod 2 \pi)
$$

We observe that in this case the maneuver (22) corresponds to invert the $z$ component of the velocity.

\subsection{Effective phases}

Looking at the central part of (8) or equivalently, if the satellite is on a Lissajous orbit we have,

$$
x(t)=A_{x} \cos (\omega t+\phi), \quad y(t)=\bar{k} A_{x} \sin (\omega t+\phi), \quad z(t)=A_{z} \cos (\nu t+\psi) .
$$

We note that due to the autonomous character of the original system of differential equations (3), we can reset $t=0$ at any time if we recompute the $A_{i}$ values of the solution (8) using equations (6) and (7). For the central part (24) mentioned above, due to the invariance of the amplitudes $A_{x}$ and $A_{z}$ it is even easier, since $t$ can be reseted to zero at time $t_{0}$ just changing the phases $\phi$ and $\psi$ by $\phi+\omega t_{0}$ and $\psi+\nu t_{0}$ respectively. This observation motivates the following definitions.

Let us define the effective phase $\Phi$ as all the epochs $t$ and all the phases $\phi$ such that $\Phi=\omega t+\phi(\bmod 2 \pi)$. In the same way we define the effective phase $\Psi$ as all the epochs $t$ and all the phases $\psi$ such that $\Psi=\nu t+\psi$ $(\bmod 2 \pi)$. Although as defined effective phases are subsets in the space $\mathbb{R} \times[0,2 \pi]$, for convenience they will be identified by the numbers $\Phi$ and $\Psi$ in $[0,2 \pi]$.

Using equations (24) and taking also into account the velocities, we note that there is a biunivocal correspondence between a pair of effective phases $(\Phi, \Psi)$ and a state $(x, y, z, \dot{x}, \dot{y}, \dot{z})$ on a Lissajous orbit of given amplitudes $A_{x}$ and $A_{z}$. In fact, from a dynamical systems point of view, this is a consequence that Lissajous orbits are 2D tori since $\Phi$ and $\Psi$ are identified $(\bmod 2 \pi)$. We are just using the well known action-angle variables of the tori.

The convenience of using the effective phases becomes clear since in the space $(\Phi, \Psi)$ a trajectory such as $(24)$ with initial phases $\phi_{i}, \psi_{i}$, is a straight line of slope $\omega / \nu$, starting at the point $\left(\phi_{i}, \psi_{i}\right)$, which propagates with constant velocity components $\omega$ and $\nu$ respectively in the directions $\Phi$ and $\Psi$. So, dynamics are much easier.

As a first application of the effective phases we have that, looking at (13) we see that the optimal maneuver to change the $A_{x}$ amplitude has to be done when the trajectory in the space of effective phases crosses either the line $\Phi=\beta+\frac{\pi}{2}$ or $\Phi=\beta+\frac{3 \pi}{2}$. Also according to (21) the optimal change of $A_{z}$ is done either when crossing $\Psi=\frac{\pi}{2}$ or $\Psi=\frac{3 \pi}{2}$. But in fact, the space of effective phases, from now on the effective phases plane (EPP), can be used as a nice and general tool for mission design. Some of its applications are presented in the following sections. 


\section{Eclipse Avoidance and LOEWE Strategies}

Usually a technical requirement for libration point satellites is to avoid an exclusion zone. For orbits about $\mathrm{L}_{1}$ in the Sun-Earth system the exclusion zone is three degrees about the solar disk as seen from Earth (see [5]). For orbits about $\mathrm{L}_{2}$ in the Sun-Earth system sometimes the Earth half-shadow has to be avoided. In both cases, since Sun and Earth are located in the $x$ axis, the exclusion zone is set as a disk in the $y z$ plane centered at the origin. Traditionally, halo orbits have been used to avoid the exclusion zone (see [5]) however they drawback is that the $y$ excursions of the satellite are very big. This fact increases the complexity and cost of some hardware parts of the satellite. Lissajous orbits suit much better in most of the cases. However, if the duration of the mission is long enough, the satellite will irremediable cross the exclusion zone. The time to enter eclipse depends on the initial phases $\phi_{i}, \psi_{i}$, and in the best case the time span between eclipses is about 6 years for an orbit of moderate size (see [7], [17]).

We have developed new eclipse avoidance strategies based on the change of phase explained in the previous section. The strategies will be named LOEWE (Lissajous Orbit Ever Without Eclipse) and they are based on single impulses. Strategies with more than one impulse could also be studied but are not the purpose of this paper. Moreover one of the nicest things of the single impulse strategies is their simplicity besides an affordable cost. As we will see, the idea is to perform the maneuver near the "corners" of the Lissajous figure corresponding to the $y z$ projection, where velocities are small. We are near these corners just when we are about to enter to or exit from an eclipse. This will provide us with maximum time without eclipse after having performed the maneuver.

Let us use the EPP to represent the LOEWE strategies. Assuming that the satellite is on a Lissajous orbit (24) of amplitudes $A_{x}$ and $A_{z}$, the exclusion zone appears in the $y z$ plane as a disk of radius $R, y^{2}+z^{2}<R^{2}$. Of course we must have $R<A_{y}=\bar{k} A_{x}$, and $R<A_{z}$. The border of the disk in the plane of effective phases satisfies the equation,

$$
\bar{k}^{2} A_{x}^{2} \sin ^{2} \Phi+A_{z}^{2} \cos ^{2} \Psi=R^{2},
$$

and are the ellipse like plots represented in figure 2. When the Lissajous trajectory, represented by a straight line of constant speed in the EPP, cuts one of these curves it means that the satellite is entering the exclusion zone (see again figure 2). In this way we note that the computation of the time when the satellite will enter the exclusion zone reduces to compute the intersection of the straight line with the first exclusion zone it hits. Thus, time is just proportional to the distance to the intersection point.
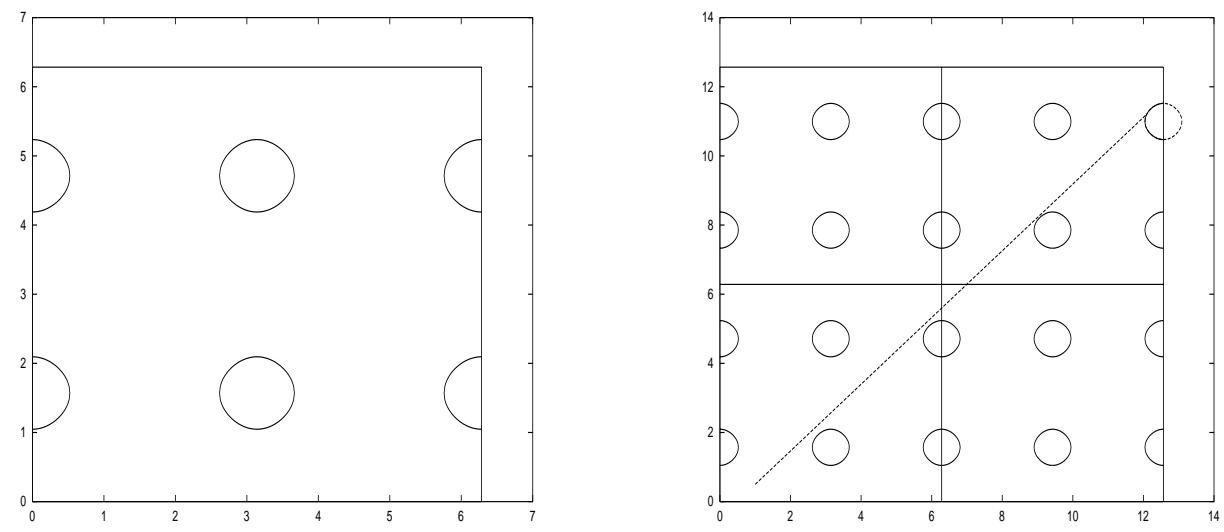

Figure 2: Exclusion zone in the effective phases plane (EPP) (left) and Lissajous trajectory hitting an exclusion zone (right). We remark that in the EPP a Lissajous trajectory appears as an straight line which propagates at a constant speed. We will always use the horizontal axis for $\Phi$ and the vertical one for $\Psi$.

For convenience, trajectories in the EPP will be reduced to $[0,2 \pi] \times[0,2 \pi]$ since, as we said, the values $\Phi=0$ and $\Phi=2 \pi$ are identified as well as $\Psi=0$ and $\Psi=2 \pi$ are. A typical LOEWE trajectory in the EPP looks like the one represented in figure 3 were the discontinuities correspond to maneuvers. We also note that a point in the EPP gives as well information about the location of the satellite with respect to the libration point. When the satellite is on a Lissajous orbit, the rectangular regions represented in figure 3 correspond to constant sign of local 


\begin{tabular}{|l|c|c|c|c|c|}
\hline & $\omega$ & $\nu$ & $\nu / \omega$ & usual $R(\mathrm{~km})$ & angle from Earth \\
\hline $\mathrm{L}_{1}$ & 2.08645356 & 2.01521066 & 0.9658545 & 90000 & $\simeq 3.5$ deg radius \\
$\mathrm{L}_{2}$ & 2.05701420 & 1.98507486 & 0.9650272 & 14000 & $\simeq 0.54$ deg radius \\
\hline
\end{tabular}

Table 1: Values for the Earth+Moon-Sun case.

coordinates. More precisely, $z>0$ in regions A,B,C,D, $y>0$ in regions $\mathrm{C}, \mathrm{D}, \mathrm{G}, \mathrm{H}$ and $x>0$ in regions $\mathrm{A}, \mathrm{D}, \mathrm{E}, \mathrm{H}$. So, due to the reference system we took in (2) the excluded region in the middle of the EPP (about $\Phi=\pi$ ) represent the exclusion zone in the Lissajous orbit when it is closer to the Earth and the ones about $\Phi=0,2 \pi$ the part when it is farther from the Earth.
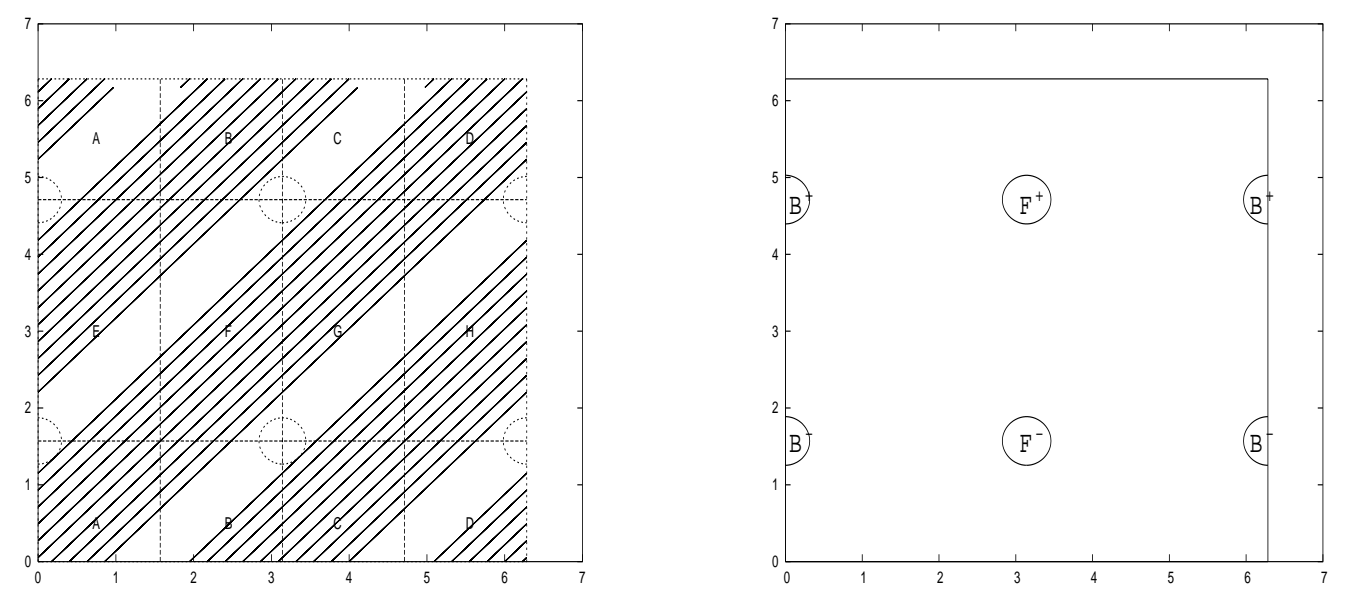

Figure 3: Left. Representation of a LOEWE trajectory in the effective phases plane. Discontinuities correspond to maneuvers. The rectangular regions labeled with capital letters, are the regions where the $x, y$ and $z$ coordinates have constant sign (see explanations in the text). Right. The meaning of the different exclusion zones in the EPP for the $\mathrm{L}_{2}$ case. The ones marked with $\mathrm{F}$ correspond to the one closest to the Earth $(x>0)$ and the ones with $\mathrm{B}$ correspond to the farthest. For the $\mathrm{L}_{1}$ case, according to (2), the letters $F$ and $B$ should be interchanged.

In table 1 we represent the values of $\omega$ and $\nu$ that we have for the Earth+Moon-Sun case, as well as the usual radius of the exclusion zone. We note that the slope of a Lissajous trajectory in the EPP is $\nu / \omega$ which in any case is slightly less than one. This means that the upper tangential trajectories to the excluded zones in the EPP as well as their neighbors are not good initial conditions, because they lead to collision in less than a year. On the other hand, initial conditions giving the lower tangential points are the best ones in terms of maximizing the time without eclipse, with more than 6 years from departure to collision (see fig 4). In most of our discussion we will consider square Lissajous. This is, $A_{y}=A_{z}$ and this value will be denoted by $A$. In this case the border of the exclusion zones (25) is given by $\sin ^{2} \Phi+\cos ^{2} \Psi=(R / A)^{2}$, meaning that the time without eclipse depends only on the relative size $R / A$ of the exclusion zones. This is, missions with different amplitudes and different exclusion zone radius have identical representations in the EPP if the relative size $R / A$ is the same. This fact allows us to compute the maximum time for a mission without hitting an exclusion zone as a function only of $R / A$ which will be considered in percentage. It is just a matter of computing the the intersection time of the orbits starting at the lower tangential conditions. In all the usual cases $(R / A$ less than $30 \%)$, the results are about 6 years and can be seen in figure 5 .

\subsection{Long term missions. The tangent to tangent cycle}

As a result of our previous discussion we use the lower tangential points or, more specifically, lower tangential trajectories in the EPP, as target points for our strategy. Thanks to the geometrical simplicity of this way of tackling the problem, we can easily compute the change of phases we need to reach one of these trajectories once 

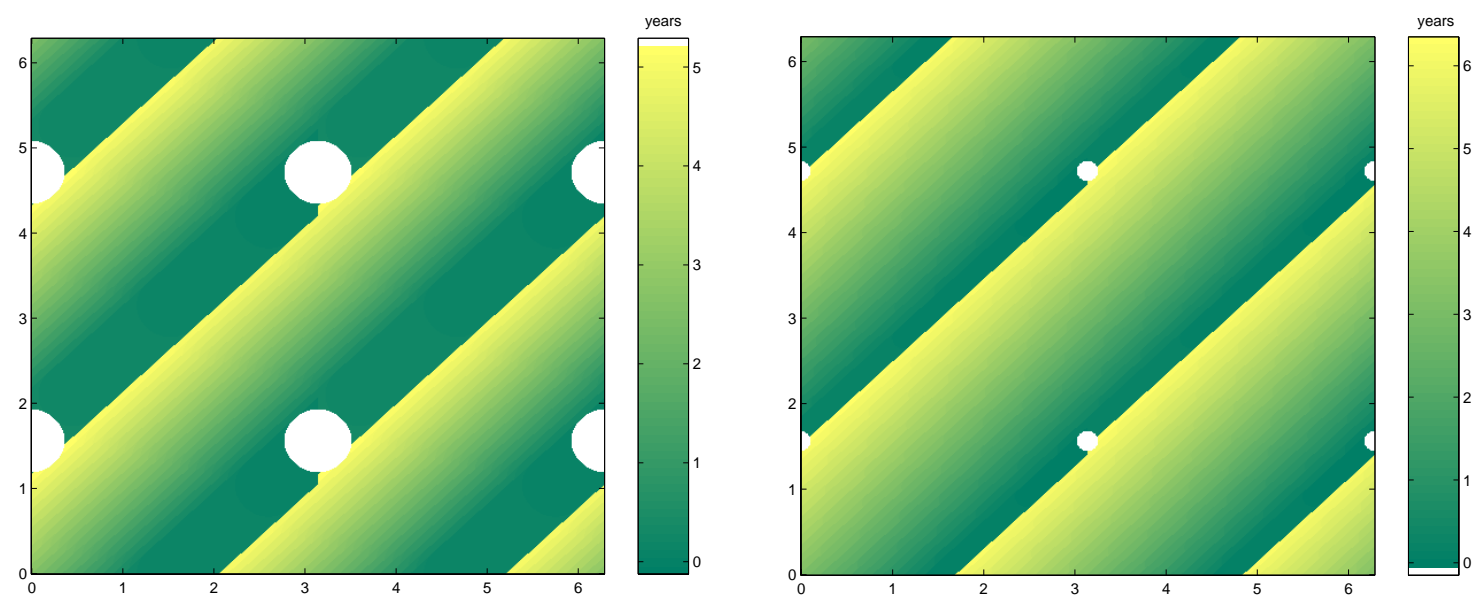

Figure 4: Initial phases classification according to the time when they first hit an exclusion zone. Right, $\mathrm{L}_{1}$ case $A_{y}=$ $A_{z}=250000 \mathrm{~km}, R=90000 \mathrm{~km}$. Left, $\mathrm{L}_{2}$ case $A_{y}=A_{z}=120000 \mathrm{~km}, R=14000 \mathrm{~km}$.
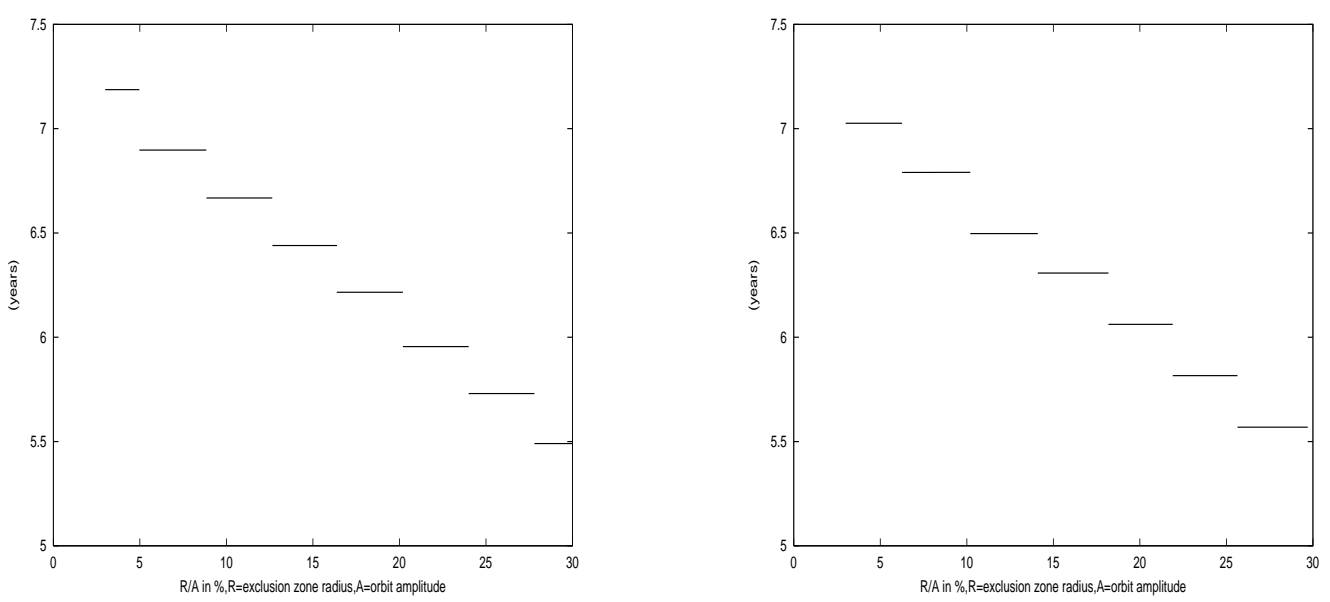

Figure 5: Maximum time without entering an exclusion zone for square Lissajous as a function of the relative size of the exclusion zone $R / A$ in percentage. Lissajous about $\mathrm{L}_{1}$ (left), about $\mathrm{L}_{2}$, (right). 
we approach the exclusion zone, avoiding much more difficult algorithms as the ones we used in [2]. The two strategies we discussed in the previous section will be considered: the $x y$-strategy given by equations (15) and (17) and the $z$-strategy given by equations (22) and (23). The basic idea comes from the fact that in the EPP the Lissajous trajectory looks like a set of parallel lines and that (17), (23) represent jumps in the $\Phi$ and $\Psi$ directions respectively. Then, for the $x y$-strategy we just have to measure the horizontal distance from the collision phases to the nearest lower tangential trajectory, whereas for the $z$-strategy, the vertical distance. But there is a difference to be remarked between both strategies.

In the case of $z$-maneuvers we know that (22) correspond to invert the sing of the $z$-velocity. Since when a $z$-maneuver is performed the satellite remains in a Lissajous orbit, and $\dot{z} \geq 0$ in the upper half part of the EPP, this type of maneuver is seen in the EPP either as a jump symmetrical with respect to the line $\Psi=\pi$ or symmetrical with respect to $\Psi=0$ (equiv. $\Psi=2 \pi$ ).

In the case of a $x y$-maneuver the situation is slightly different since we must take into account that a maneuver of the type (9) transfers the satellite from a Lissajous orbit to the stable manifold of another Lissajous orbit. There is a short time span after a maneuver where the trajectory has stable hyperbolic exponentially decreasing terms that cannot be neglected when comparing the trajectory to a Lissajous one. During this time span, the representation of the trajectory in the EPP gives only an approximate idea (although good enough) about the actual position of the satellite. There could be rare exceptions that, when the trajectory in the EPP looks tangent to the exclusion zone, in fact the satellite still had some stable manifold component producing a deviation with respect to the represented coordinates, in the wrong direction, and the satellite touched the exclusion zone. These exceptions could be easily avoided just considering the exclusion zone slightly bigger than what is strictly necessary, although we must say that in all the examples and simulations of this paper there has been no need of the enlargement of the exclusion zone. Apart from this remark, in a similar way as the $z$-maneuvers, the $x y$-maneuvers appear as jumps symmetrical with respect to $\Phi=\beta$ or with respect to $\Phi=\pi+\beta$. Needless to say that both maneuvers can also be computed by implementing an easy algorithm to find the suitable $t_{m}$, which using (17) or (23) obtains the required $\Delta \Phi=\phi_{f}-\phi_{i}$ or $\Delta \Psi=\psi_{f}-\psi_{i}$. In figure 6 we represent the way how these two strategies work. See [2] for other special symmetries of the $x y$ and $z$-maneuvers when performed in certain locations.
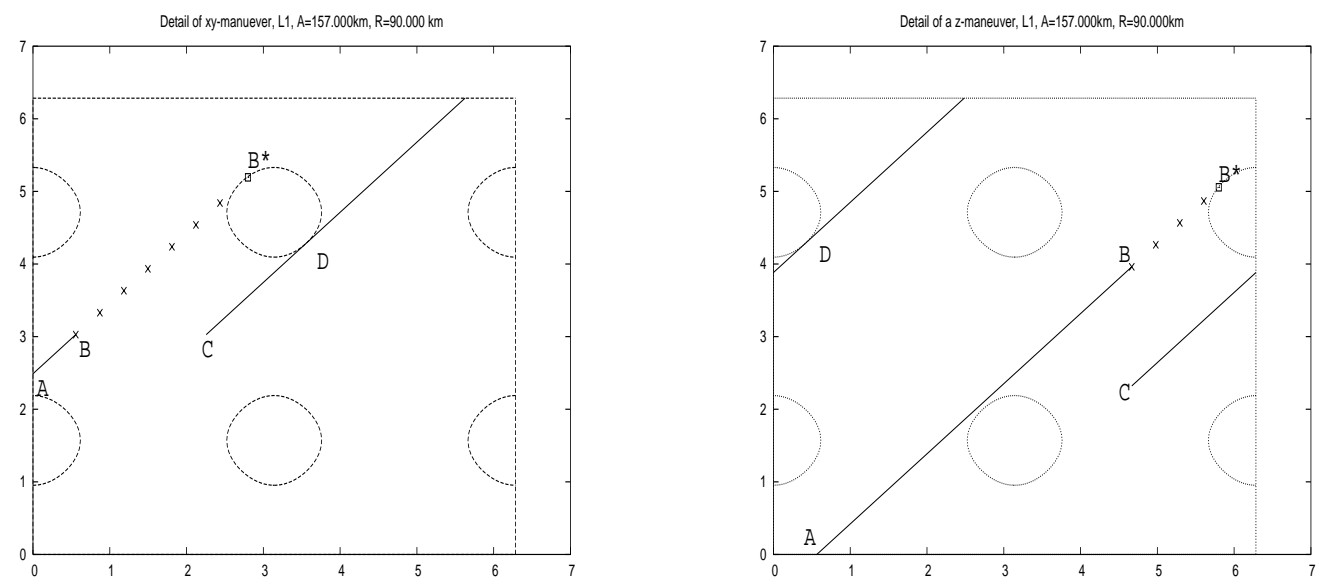

Figure 6: Detail of $x y$ (left) and $z$ (right) maneuvers for the LOEWE strategy. Let us assume that the Lissajous trajectory starts at A. In both cases the maneuver is computed to reach a lower tangential trajectory to an exclusion zone before a collision would happen in $\mathrm{B}^{*}$. The result of the maneuver is to jump from B to C in the EPP. Note that in these cases the jump of the $x y$-maneuver is symmetrical with respect to $\Phi=\beta$ and the one for the $z$-maneuver is symmetrical with respect to $\Psi=\pi$. Lower tangency to the exclusion zone occurs at point $\mathrm{D}$.

The LOEWE strategy consists in making the satellite to enter and follow a tangent to tangent cycle. Let us assume that the satellite is injected into the Lissajous trajectory at the point $\left(\Phi_{i}, \Psi_{i}\right)$ of the EPP. Take this point as time $t=0$. As time increases, the satellite approaches an exclusion zone which would be intersected at time $t_{c}$. Prior to the collision, a time $t_{m}$ is selected in order to apply an $x y$-maneuver (i.e. a jump $\Delta \Phi$ ) or a $z$-maneuver (i.e. a jump $\Delta \Psi)$ in such a way that the point $\left(\Phi_{m}, \Psi_{m}\right)$ at time $t_{m}$ in the EPP jumps to a new one $\left(\Phi_{t g}, \Psi_{t g}\right)$ 
(equal to $\left(\Phi_{m}+\Delta \Phi, \Psi_{m}\right)$ or to $\left.\left(\Phi_{m}, \Psi_{m}+\Delta \Psi\right)\right)$ which goes into a lower tangential trajectory to the exclusion zone as it is represented in figure 6 . Then, the tangent to tangent cycle starts in the natural way by renaming $\left(\Phi_{t g}, \Psi_{t g}\right)$ to $\left(\Phi_{i}, \Psi_{i}\right)$ and reseting time to zero. Once in the cycle, after each maneuver the satellite will be about six years free of eclipse, as shown in figure 5 ,

There is a technical remark to be made about closing a cycle. We define a cycle as the part of trajectory in the EPP comprised between two lower tangencies and having a maneuver in between. However, several slightly different patterns in the $x y z$ representation of the trajectory can appear. This is due to the fact that the exclusion zones displayed in the EPP have different meanings (see figure 3). Let us assume that the cycle begins with a tangency to the exclusion zone $\mathrm{F}^{+}$(the exclusion zone closer to the Earth), next tangency closing the cycle in the EPP can only happen either with $\mathrm{F}^{-}$(again the exclusion zone closer to the Earth), or with $B^{+}$(the exclusion zone farther from Earth). In general, a cycle goes from $\mathrm{F}^{+,-}$(resp. from $\mathrm{B}^{+,-}$) to $\mathrm{F}^{-,+}$or to $\mathrm{B}^{+,-}$(resp. to $\mathrm{B}^{-,+}$ or to $\mathrm{F}^{+,-}$). If the cycle ends with the same letter as it started (i.e. from $\mathrm{F}$ to $\mathrm{F}$ or from $\mathrm{B}$ to $\mathrm{B}$ ) we say that the cycle is one-sided, otherwise we say that is two-sided. Moreover, for both cases two cycles are needed to repeat the same starting position and velocity (and so the same pattern in the $x y z$-coordinates), but since both maneuvers of these cycles have the same magnitude and the time span between them is the same, it is not necessary to make such distinctions for the total amount of delta-v expended and for the results we present. Only to say that the fact that for a given Lissajous, its LOEWE strategy is associated to a one sided or to a two-sided cycles, depends exclusively on the size of $R / A$ i.e. the size of the exclusion zone in the EPP, since the Lissajous trajectories in the EPP are always seen as parallel lines separated by a constant distance. Figures 7 and 8 give some examples of LOEWE strategies associated to one-sided or two-sided cycles. Note that for one-sided cycles the projection of the maneuvers in the $x y$ plane is always the same.

\subsubsection{Summary of results for the tangent to tangent cycle}

All Lissajous orbits considered have amplitude $A=A_{y}=A_{z}$ ("square Lissajous"). Qualitatively, the results depend only on the relative amplitude R/A. However, in terms of real cost, they also depend on A. The main result is that the $x y$-strategy is proportionally a little cheaper for orbits of relative size R/A less than $60 \%$. When $\mathrm{R} / \mathrm{A}$ is bigger than $60 \%$ then the $z$-strategy is cheaper than $x y$-strategy (see figure 9 ). Concerning the actual costs for the LOEWE strategies, these can be seen in table 2 for the case about $\mathrm{L}_{1}$ and in table 3 for the case about $\mathrm{L}_{2}$. Fixing the exclusion zone to $R=90000 \mathrm{~km}$ for the case of $\mathrm{L}_{1}$ and to $R=14000 \mathrm{~km}$ for the case of $\mathrm{L}_{2}$ we obtain the figures of the average cost per year with respect to the amplitude selected displayed in figure 10. See also [2] for rough and preliminary analytical estimations.

It comes as a bit of a surprise that the results obtained are only partially linear. For example, for $\mathrm{R}=14000 \mathrm{~km}$ and $A=77600 \mathrm{~km}$ about $\mathrm{L}_{2}$, we have an average cost of $2.5 \mathrm{~m} / \mathrm{s}$ per year in the cycle, while $A=77700 \mathrm{~km}$ costs $1.9 \mathrm{~m} / \mathrm{s}$ each year. We can clearly distinguish 'groups' of relative amplitudes, for which the cycles have the same period and the $\Delta \phi, \Delta \psi$ jumps are also the same. In these groups the cost is not proportional to $R / A$, but to the real amplitude of the Lissajous, $A$. The reason for these discontinuities is the following. The LOEWE cycle essentially consists in performing the cheapest change in the effective phases in such a way to repeat the tangent condition. Since Lissajous trajectories in the EPP appear as parallel lines separated by a constant amount, what we get is a discreet set of possible jumps. If the amplitude $A$ decreases continuously, the relative size of the exclusion zone appears bigger in the EPP. So an amplitude will be reached which will oblige a larger change of phases to be executed. Even though the relative amplitude can be continuously varied, the size of the possible jumps cannot, as they belong to a discreet set. This is the reason for the discontinuities in tables 2 and 3 and in figure 10. It also provides us with an explanation for the fact that in each of the groups the cost is proportional to the amplitude (the smaller the orbit, the cheaper the maneuver) but, globally, the cost decreases as the amplitudes become larger. Finally, in figure 11 the time to execute the maneuver prior entering the exclusion zone is shown. For the usual exclusion zones and amplitudes is about 40 or 50 days depending on the strategy.

\subsubsection{Some comments on non-square Lissajous}

We say that the Lissajous is non-square when $A_{y} \neq A_{z}$. In the effective phases plane the exclusion zones are no longer similar to disks, but to ellipses. If $A_{y}$ is greater than $A_{z}$ the horizontal diameter is shorter than the vertical. Consequently, differences in cost between executing $x y$-maneuvers or $z$-maneuvers become more significant as the difference between the two amplitudes increases. 

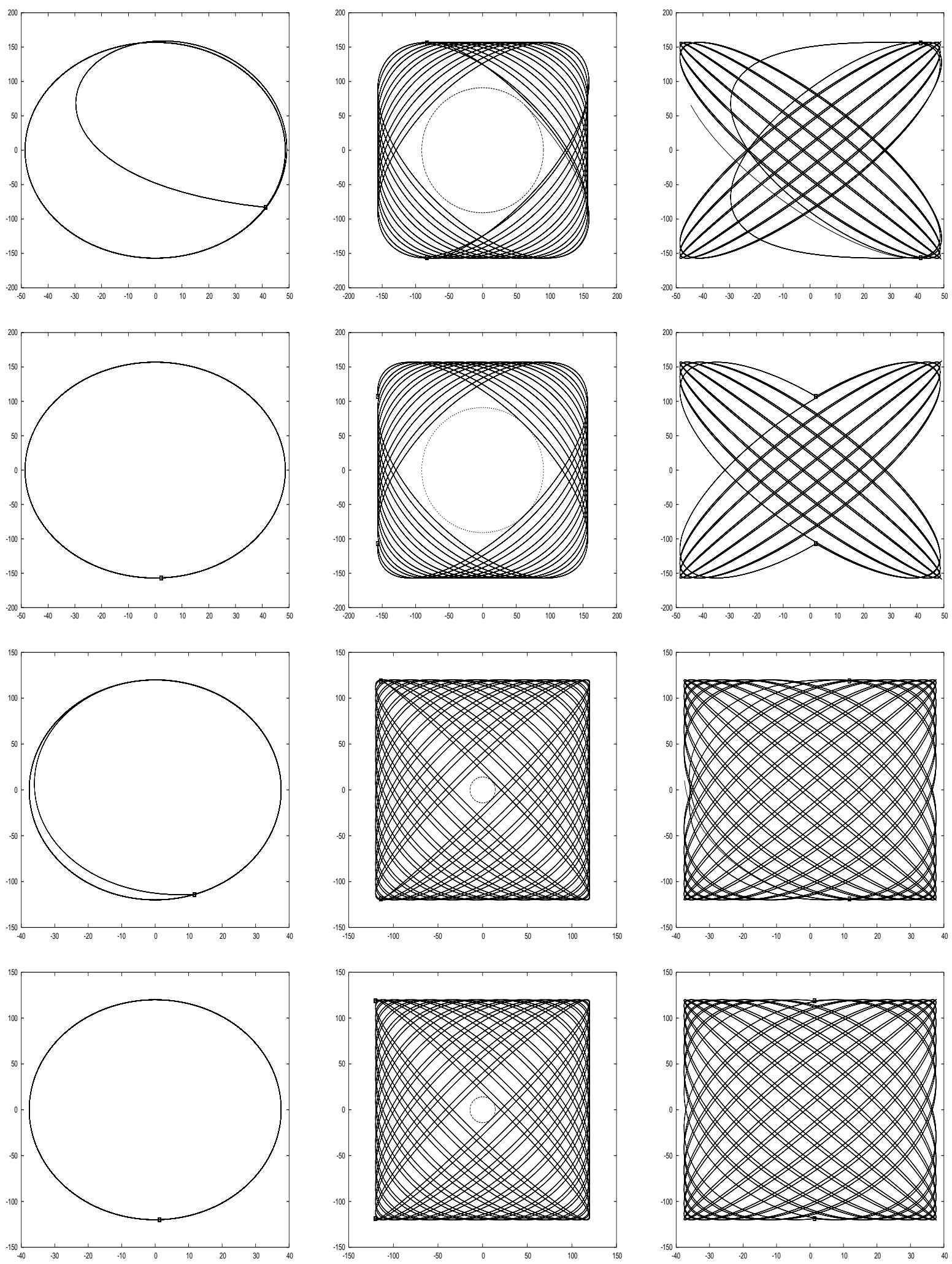

Figure 7: Examples of one-sided cycles. Top two rows, trajectories about $\mathrm{L}_{1}$ with $A=157000 \mathrm{~km}, R=90000 \mathrm{~km}$. Bottom two rows, trajectories about $\mathrm{L}_{2}$ with $A=120000 \mathrm{~km}, \mathrm{R}=14000 \mathrm{~km} . x y$-maneuvers are used in rows 1,3 and $z$ maneuvers in rows 2 and 4 . From left to right displaying the $x y, y z$ and $x z$ projections. The maneuvers are marked with a small box. 

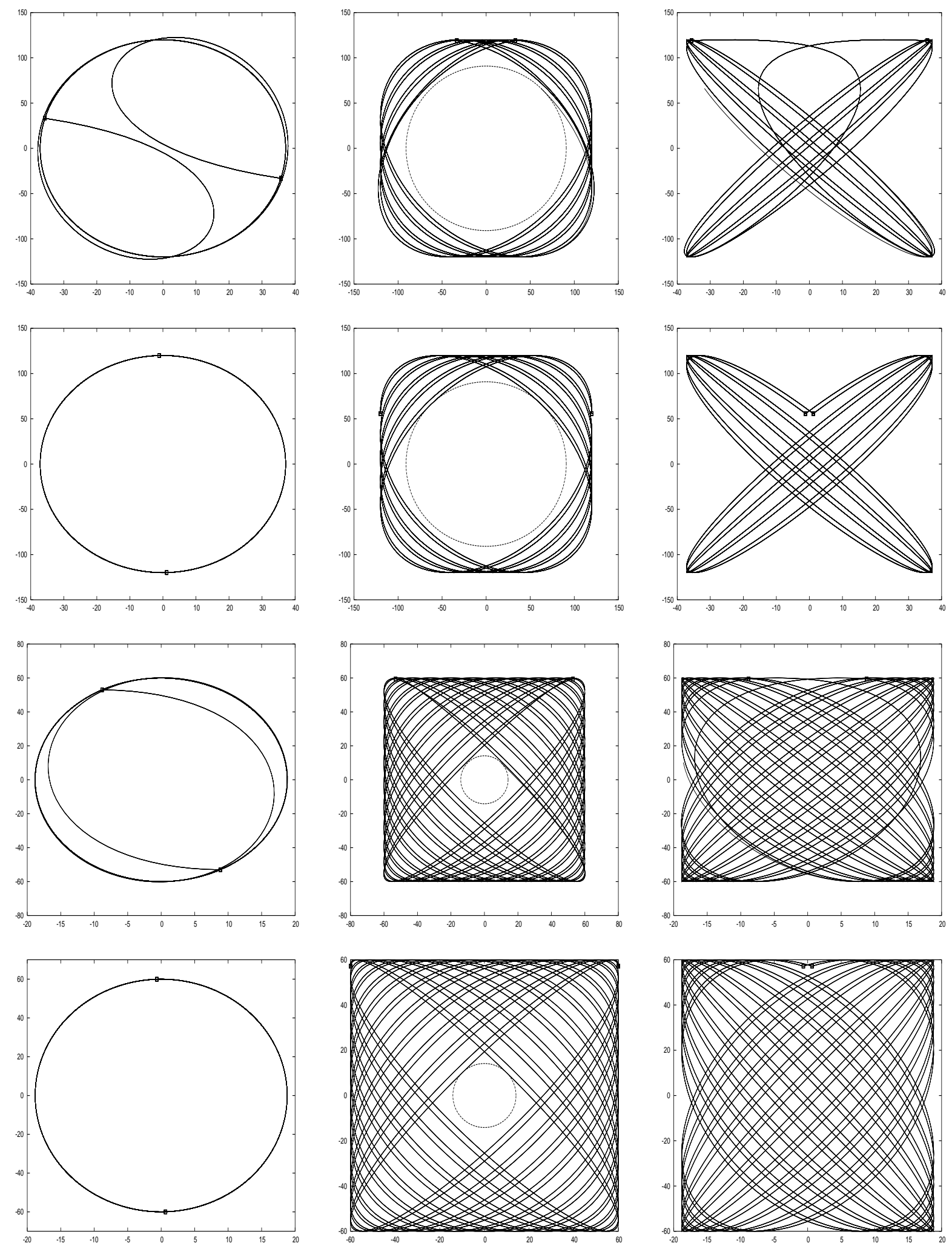

Figure 8: Examples of two-sided cycles. Top two rows, trajectories about $\mathrm{L}_{1}$ with $A=120000 \mathrm{~km}, R=90000 \mathrm{~km}$. Bottom two rows, trajectories about $\mathrm{L}_{2}$ with $A=60000 \mathrm{~km}, \mathrm{R}=14000 \mathrm{~km} . x y$-maneuvers are used in rows 1,3 and $z$ maneuvers in rows 2 and 4 . From left to right displaying the $x y, y z$ and $x z$ projections. The maneuvers are marked with a small box. 

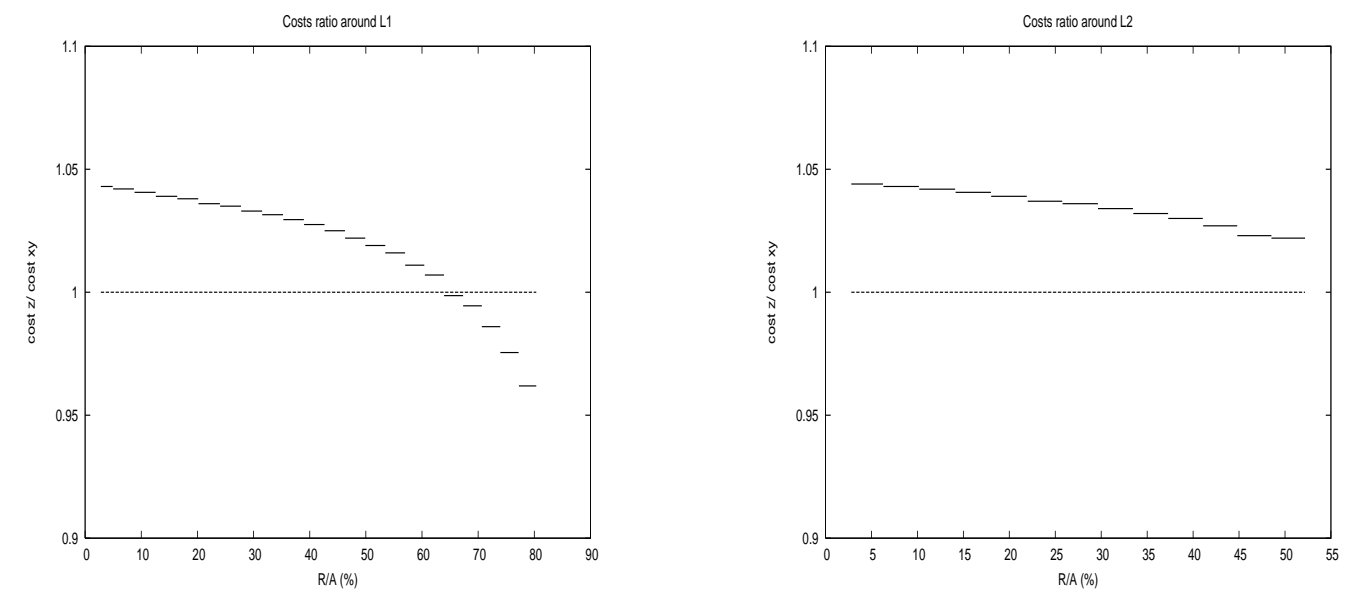

Figure 9: Ratio $\frac{z-\cos t}{x y-\operatorname{cost}}$ for the $\mathrm{L}_{1}$ case (left) and for the $\mathrm{L}_{2}$ case (right).

\begin{tabular}{|cc|cc|cc|cc|}
\hline \multicolumn{2}{|c|}{$\mathrm{R} / \mathrm{A}(\%)$} & \multicolumn{2}{|c|}{$\mathrm{ACY}=K_{*} \times A(\mathrm{~cm} / \mathrm{s})$} & \multicolumn{2}{c|}{ TWE (years) } & \multicolumn{2}{c|}{$\mathrm{MC}=M_{*} \times A(\mathrm{~cm} / \mathrm{s})$} \\
\hline From & To & $K_{x y}$ & $K_{z}$ & $x y$ & $z$ & $M_{x y}$ & $M_{z}$ \\
\hline 77.25 & 80.35 & 39.571 & 38.063 & 1.737 & 1.917 & 68.727 & 72.972 \\
73.95 & 77.20 & 33.785 & 32.955 & 1.985 & 2.157 & 67.059 & 71.077 \\
70.66 & 73.94 & 29.191 & 28.784 & 2.233 & 2.396 & 65.184 & 68.978 \\
67.32 & 70.65 & 25.435 & 25.295 & 2.481 & 2.636 & 63.108 & 66.680 \\
63.92 & 67.31 & 22.291 & 22.322 & 2.729 & 2.876 & 60.838 & 64.191 \\
60.50 & 63.92 & 19.608 & 19.746 & 2.977 & 3.115 & 58.379 & 61.517 \\
57.00 & 60.45 & 17.282 & 17.486 & 3.225 & 3.355 & 55.741 & 58.666 \\
53.46 & 56.99 & 15.238 & 15.480 & 3.473 & 3.595 & 52.932 & 55.647 \\
49.90 & 53.45 & 13.425 & 13.684 & 3.722 & 3.834 & 49.958 & 52.467 \\
46.30 & 49.89 & 11.797 & 12.061 & 3.970 & 4.074 & 46.831 & 49.136 \\
42.65 & 46.28 & 10.327 & 10.586 & 4.218 & 4.313 & 43.560 & 45.665 \\
39.00 & 42.64 & 8.991 & 9.238 & 4.466 & 4.553 & 40.154 & 42.061 \\
35.30 & 38.96 & 7.769 & 7.999 & 4.714 & 4.793 & 36.624 & 38.337 \\
31.54 & 35.25 & 6.646 & 6.856 & 4.962 & 5.032 & 32.982 & 34.502 \\
27.80 & 31.53 & 5.611 & 5.798 & 5.210 & 5.272 & 29.238 & 30.569 \\
24.05 & 27.75 & 4.654 & 4.816 & 5.458 & 5.512 & 25.403 & 26.547 \\
20.20 & 24.00 & 3.766 & 3.903 & 5.706 & 5.751 & 21.491 & 22.450 \\
16.41 & 20.15 & 2.941 & 3.052 & 5.955 & 5.991 & 17.512 & 18.287 \\
12.60 & 16.40 & 2.173 & 2.258 & 6.202 & 6.231 & 13.479 & 14.072 \\
8.80 & 12.55 & 1.458 & 1.517 & 6.451 & 6.470 & 9.405 & 9.816 \\
5.00 & 8.77 & 0.791 & 0.824 & 6.699 & 6.710 & 5.301 & 5.533 \\
2.80 & 4.93 & 0.170 & 0.177 & 6.947 & 6.949 & 2.363 & 1.233 \\
\hline
\end{tabular}

Table 2: Summary of results for avoiding the exclusion zone about $\mathrm{L}_{1}$ using LOEWE strategies with $x y$ or $z$ maneuvers. $\mathrm{ACY}=K_{*} \times A$, is the average cost per year. TWE is the time without eclipse once a maneuver is performed and, $\mathrm{MC}=M_{*} \times A$, is the cost of each maneuver in the cycle. In the formulae, the amplitude $A$ is considered to be in thousands of $\mathrm{km}$. 


\begin{tabular}{|cc|cc|cc|cc|}
\hline \multicolumn{2}{|c|}{$\mathrm{R} / \mathrm{A}(\%)$} & \multicolumn{2}{|c|}{$\mathrm{ACY}=K_{*} \times A(\mathrm{~cm} / \mathrm{s})$} & \multicolumn{2}{|c|}{ TWE (years) } & \multicolumn{2}{c|}{$\mathrm{MC}=M_{*} \times A(\mathrm{~cm} / \mathrm{s})$} \\
\hline From & To & $K_{x y}$ & $K_{z}$ & $x y$ & $z$ & $M_{x y}$ & $M_{z}$ \\
\hline 48.53 & 52.18 & 12.688 & 12.966 & 3.778 & 3.889 & 47.939 & 50.428 \\
44.81 & 48.52 & 11.099 & 11.376 & 4.030 & 4.132 & 44.732 & 47.009 \\
41.10 & 44.80 & 9.664 & 9.931 & 4.282 & 4.375 & 41.380 & 43.449 \\
37.30 & 41.05 & 8.358 & 8.609 & 4.534 & 4.618 & 37.894 & 39.758 \\
33.50 & 37.28 & 7.164 & 7.394 & 4.786 & 4.861 & 34.286 & 35.947 \\
29.65 & 33.45 & 6.067 & 6.274 & 5.037 & 5.104 & 30.566 & 32.027 \\
25.80 & 29.64 & 5.056 & 5.260 & 5.289 & 5.347 & 26.747 & 28.021 \\
22.00 & 25.75 & 4.122 & 4.276 & 5.541 & 5.591 & 22.842 & 23.905 \\
18.02 & 21.90 & 3.256 & 3.380 & 5.793 & 5.834 & 18.862 & 19.717 \\
14.15 & 18.00 & 2.452 & 2.551 & 6.045 & 6.077 & 14.821 & 15.504 \\
10.20 & 14.10 & 1.704 & 1.776 & 6.297 & 6.320 & 10.733 & 11.224 \\
6.30 & 10.15 & 1.009 & 1.053 & 6.549 & 6.563 & 6.610 & 6.910 \\
2.80 & 6.26 & 0.362 & 0.379 & 6.801 & 6.806 & 2.465 & 2.577 \\
\hline
\end{tabular}

Table 3: Summary of results for avoiding the exclusion zone about $\mathrm{L}_{2}$ using LOEWE strategies with $x y$ or $z$ maneuvers. Same comments of table 2 apply here.
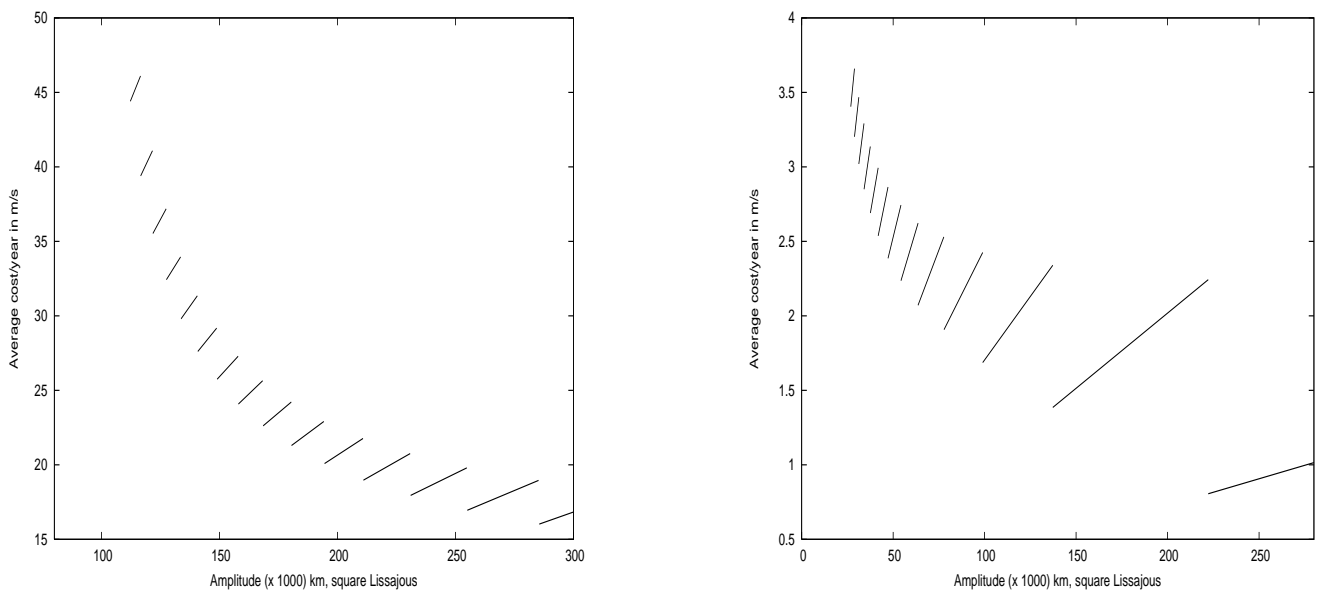

Figure 10: LOEWE average cost in $\mathrm{m} / \mathrm{s}$ per year with respect to the amplitude for a fixed exclusion zone. Left, about $\mathrm{L}_{1}$ of $R=90000 \mathrm{~km}$. Right, about $\mathrm{L}_{2}$ of $R=14000 \mathrm{~km}$. 

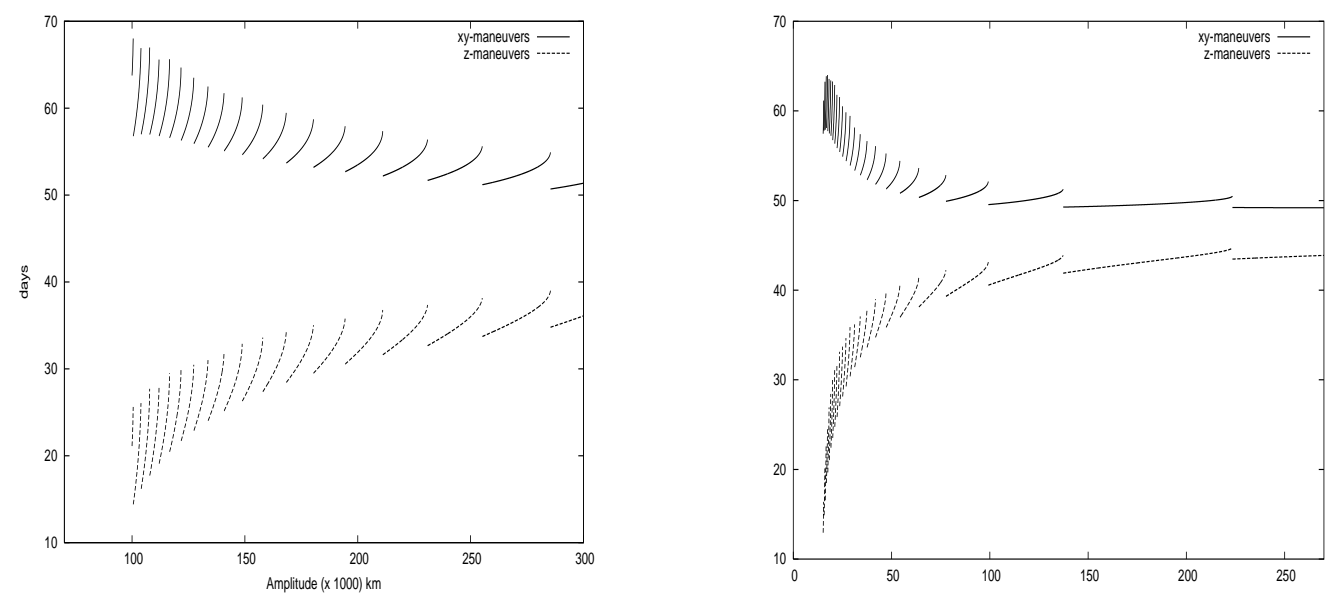

Figure 11: As a function of the amplitude, days prior entering the exclusion zone when the LOEWE $x y$ or $z$-maneuver has to be performed in oder to avoid it. Left, about $\mathrm{L}_{1}$ and exclusion zone of $R=90000 \mathrm{~km}$. Right, about $\mathrm{L}_{2}$ and exclusion zone of $R=14000 \mathrm{~km}$.

As a result of the computations, the cost of the maneuvers depends on the amplitude, being directly proportional to it (in each of the groups). So, intuitively, if for example $A_{y}$ was smaller than $A_{z}$, it would be cheaper to perform $x y$-maneuvers than $z$-maneuvers, and the other way round. We can assert this hypothesis by applying our strategy to non-square Lissajous. Furthermore, if $A_{z}=\kappa A_{y}$ the relation between costs can be written,

$$
\operatorname{cost}_{\mathrm{z}} \simeq \Upsilon \kappa \operatorname{cost}_{\mathrm{xy}}
$$

where $\Upsilon \in[0.99,1.04]$, (it is approximately 1 ). If for example $\kappa$ is less than 1 , we use z-maneuvers. The satellite
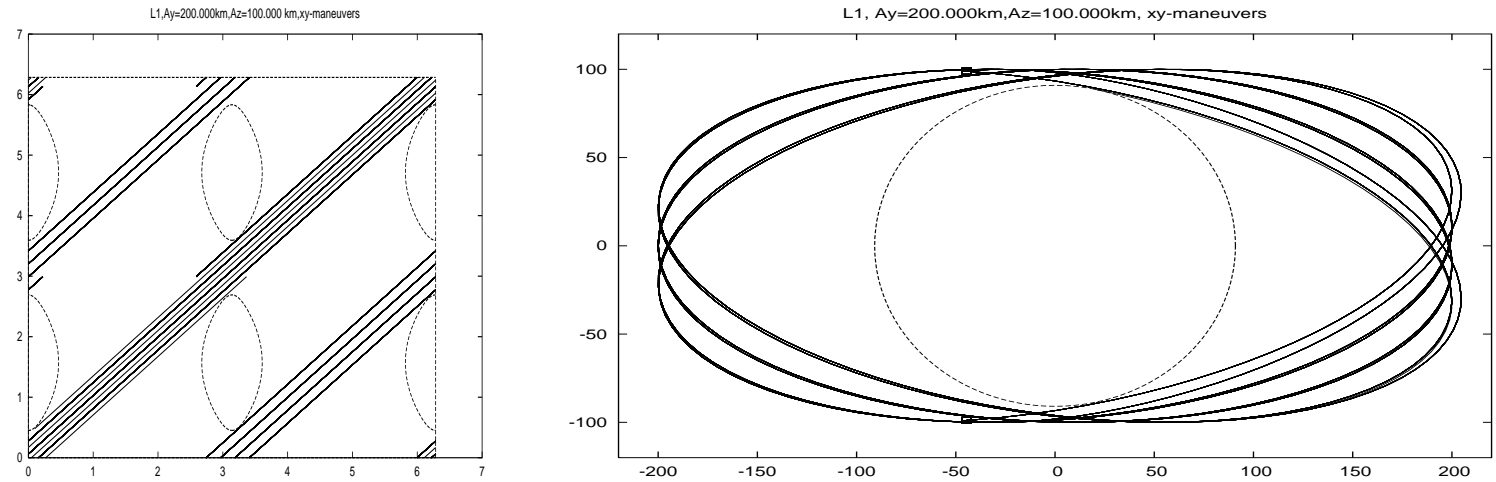

Figure 12: EPP representation an $y z$-projection of a LOEWE strategy applied to a non-square Lissajous around $\mathrm{L}_{1}$. In this case $\kappa=0.5$ and $A_{y}=200000 \mathrm{~km}$. Maneuvers in the $y z$-projection are marked with a small box.

also enters a cyclic trajectory, as in the square case. The period of the cycles coincides with one of the periods in the previous tables (although it has nothing to do with the relative amplitude). If we multiply the amplitude in the direction we are performing the maneuvers by the corresponding coefficient in the table (the one in the row we have found the period coincidence), we can compute the cost/year in the cycle with no need to compute new tables, in spite of the Lissajous being non-square. 


\subsection{Alternatives for short-term spatial missions}

The first thing to be taken into account regarding spatial missions which last for less than 7 years, is that choosing good injection phases can lead to not having to perform any maneuver. For example, if we are interested in maintaining a satellite around $\mathrm{L}_{1}$ avoiding the exclusion zone for about 4 or 5 years, we can study the regions in figure 4 for which the first collision occurs more than 5 years after the injection. However, if for some reason this ideal phases can't be reached, or the mission is longer than the maximum possible time without eclipse (see fig 5), at least one maneuver to avoid the exclusion zone is required. How do we choose the time for this maneuver?

The LOEWE strategy based on aiming at tangential trajectories in the EPP is good for long term missions, because it is cheap, gives an easy way of maintaining the satellite in a cycle and maximizes the time without eclipse. Although it would be affordable in most of the practical scenarios, it may not be the optimum strategy (in cost) amongst those that provide enough time without eclipse for a short mission.

An alternative way of choosing a good time, $t_{m}$, for the maneuver to avoid the exclusion zone is to consider a certain time interval before the predicted collision time, say a year, and for each $\tau$ in this time interval to compute,

- $t_{c}(\tau)$, the time span until next collision if we perform a maneuver at time $\tau$.

- The cost of performing the maneuver at time $\tau$.

If we select the moments at which the maneuver would provide enough time without eclipse for the mission to be successfully completed, this problem becomes an easy optimization problem: among the suitable moments for the maneuver (in terms of time), we have to select the cheapest one. Thanks to the way of tackling the orbits in the EPP, the computation of the solution of this problem is almost trivial and immediate in terms of time consumption since we only need to do an easy plot. For example, let us consider a mission on a trajectory around $\mathrm{L}_{2}$ with $\mathrm{A}=120000 \mathrm{~km}$ and $\mathrm{R}=14000 \mathrm{~km}$, which needs 10 years without eclipse and the injection phases are an almost exact lower tangential point of the exclusion zone. The first eclipse will take place 6.3 years after the injection, if no maneuver is performed. The costs using the $x y$-maneuvers and next collision times for each $\tau \in[5.3,6.3]$ are represented in figure 13 (left). But we need that $t_{m}+t_{c}\left(t_{m}\right) \geq 10$ (years). So the suitable times and costs are those represented in figure 13 (right), and we would choose one of the cheapest (which are marked with a ${ }^{\prime}{ }^{\prime}$ ).
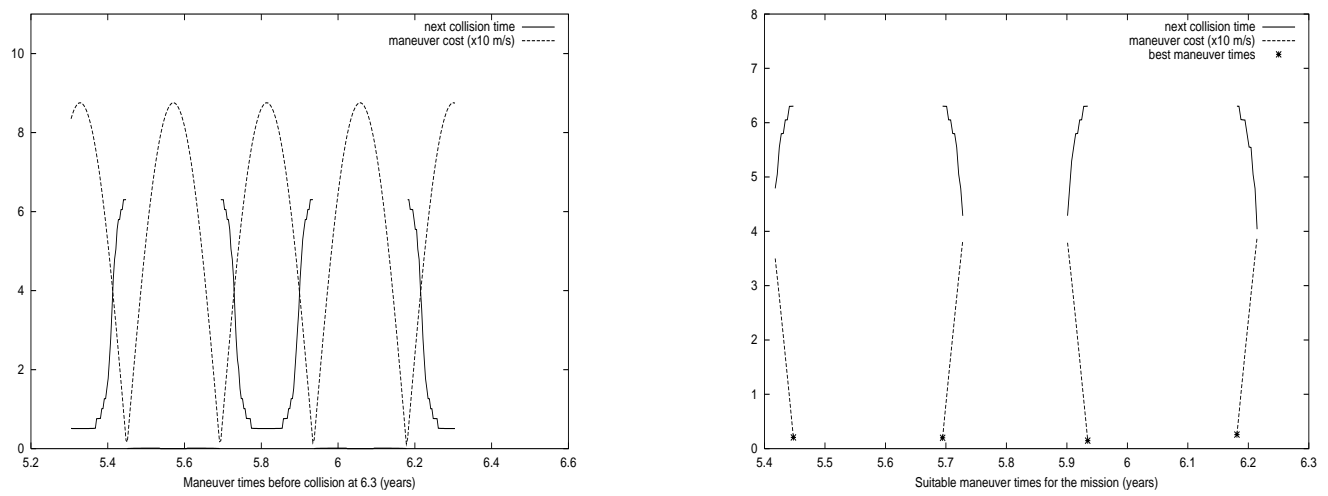

Figure 13: Left, possible $t_{m}$ with their next collision times and costs using $x y$-maneuvers. Right, Collision time and costs for the maneuver times which will allow the mission to finish without eclipse for 10 years.

Similar strategies for short term missions have been developed previously. For instance, a nice ones by Heppenheimer and Pernicka and Howell designed for trajectories around $\mathrm{L}_{1}$ with a fixed duration (see [10], [17]). In short, they divide the problem in three parts: segment 1,2 and 3 . In segment 1 and 3 , no maneuver is executed: the fact that some parts of the Lissajous can be followed without eclipse is taken advantage of. In segment 2 an eclipse avoidance strategy is implemented using $z$-maneuvers. The basic idea is to make the satellite describe almost circular revolutions about the exclusion zone, and the way to make the Lissajous revolutions become circular is to match the $x y$ and $z$ periods. For this purpose, if the satellite jumps in the $\mathrm{z}$ direction velocity twice in each revolution, it "skips" a definite amount of time. This is, a change in the phases yields an alteration of the natural velocity at which the Lissajous evolves, and so it is the same as "missing"' an interval of time (i.e. $\Delta t=\Delta \psi / \nu$ ). 
As we have seen, phase-change maneuvers which maintain the amplitude have one degree of freedom. Either the time of the maneuver, or the phase-jump it implies can be chosen. However, once one of them is fixed, there is only one possible maneuver. Pernicka and Howell use this, as well as the knowledge of the total $\Delta \psi$ needed to balance the periods, to write a cost function and minimize the $\Delta v$ expenditure in two maneuvers per revolution. As an observation, this strategy also has a geometrical interpretation in the EPP. The two maneuvers that minimize the cost per revolution are identical and result in a $\Psi$-jump which corresponds to half the vertical distance between two consecutive straight lines of the trajectory in the EPP. Thus, what the satellite does is to jump from one straight line to a previous one in every revolution (see figure 14) just before entering the exclusion zone. The cost of each maneuver is very low and proportional to the Lissajous amplitude (assuming square-Lissajous). However, one of the main drawbacks of the strategy is that it requires a great deal of maneuvers if the mission is long; aproximately 4 maneuvers each year have to be planned.

Using this strategy in the long run and measuring the amplitude, A, in thousands of $\mathrm{km}$, the cost of each maneuver is $4.3 \times A \mathrm{~cm} / \mathrm{s}$ with an average cost per year about $17.9 \times A \mathrm{~cm} / \mathrm{s}$ with maneuvers approximately every 88 days both about $\mathrm{L}_{1}$ and about $\mathrm{L}_{2}$. We note that since the strategy consists in jumping upwards in the $\Psi$ direction in the EPP, the cost only depends on the amplitude, but not on the size of the exclusion zone. Comparing these costs with the results of the LOEWE strategy (see tables 2 and 3) we clearly see that when the relative amplitude is not too big (less than 60\%), LOEWE strategy is cheaper, especially if the mission is long or if, at least, the maneuver to enter the cycle is not very expensive. That is, when the cost in the cycles is what rules over the cost before the cycles. When the exclusion zone is more than $2 / 3$ of the Lissajous amplitude, it becomes difficult to jump over the exclusion zones in the EPP. Nevertheless, the Howell and Pernicka strategy is good, because it avoids the disks by performing a lot of little maneuvers, whose costs, when added, do not exceed the cost of the big maneuver that has to be performed in the LOEWE strategy.

\section{Changing the Amplitudes}

Using (12) and (13)we get simple expressions for the minimum cost to change the in-plane amplitude $A_{y}$,

$$
\operatorname{cost}_{L_{1}}^{y}=0.37134\left|A_{y}^{(f)}-A_{y}^{i)}\right|, \quad \operatorname{cost}_{L_{2}}^{y}=0.36480\left|A_{y}^{(f)}-A_{y}^{i)}\right|,
$$

where the cost is given in meters per second and the amplitudes in thousands of $\mathrm{km}$. In the same way (20) and (21) provide the equivalent expressions for changing the out-of-plane amplitude $A_{z}$,

$$
\operatorname{cost}_{L_{1}}^{z}=0.40123\left|A_{z}^{(f)}-A_{z}^{(i)}\right|, \quad \operatorname{cost}_{L_{2}}^{z}=0.39523\left|A_{z}^{(f)}-A_{z}^{(i)}\right| .
$$

So, if we need to change from square Lissajous to square Lissajous $\left(A_{y}=A_{z}=A\right)$, an expression for the cost can be written as,

$$
\operatorname{cost}_{L_{1}}=0.77257\left|A^{(f)}-A^{(i)}\right|, \quad \operatorname{cost}_{L_{2}}=0.76003\left|A^{(f)}-A^{(i)}\right| .
$$

in case that we perform two maneuvers. The in-plane one at its optimal time (13), this is, when the trajectory seen in the EPP crosses either the line $\Phi=\beta+\frac{\pi}{2}$ or $\Phi=\beta+\frac{3 \pi}{2}$, and the out-of-plane one also at its optimal time (21), this is, when the trajectory seen in the EPP crosses either $\Psi=\frac{\pi}{2}$ or $\Psi=\frac{3 \pi}{2}$. Since these optimal times usually are very different, it is natural to wonder whether a single combined maneuver at a certain time $\tau$ would improve the results because of the parallelogram rule for the addition of vectors. This is, $C_{\text {combined }}^{\tau}=C_{x y+z}^{\tau} \leq C_{x y}^{\tau}+C_{z}^{\tau}$, where $C^{t}$ is the cost of performing a maneuver at time $t$. Denoting by $C_{s}$ the minimum possible cost for a change of amplitude of a square Lissajous using separate maneuvers, this is, the sum of the costs obtained above for single maneuvers in each direction and performed at their optimum locations, and $C_{c}^{t}$ the cost of a combined maneuver at time $t$, then, by an improvement of cost we mean to find suitable $\tau$ such that $C_{c}^{\tau} \leq C_{s}$. In this case, the percentage of improvement at time $\tau$ will be computed as

$$
I(\tau)=100 \times \frac{C_{s}-C_{c}^{\tau}}{C_{s}} .
$$

Obviously, the maximum of this improvement is achieved in each one of the four intersections between $\Phi=\beta+\frac{3 \pi}{2}$, $\Phi=\beta+\frac{\pi}{2}$, and $\Psi=\frac{\pi}{2}, \Psi=\frac{3 \pi}{2}$. So, the upper bounds for $I(\tau)$, computed at these intersections, are,

$$
\max _{L_{1}} I(\tau)=29.23642 \%, \quad \max _{L_{2}} I(\tau)=29.23266 \% \text {. }
$$



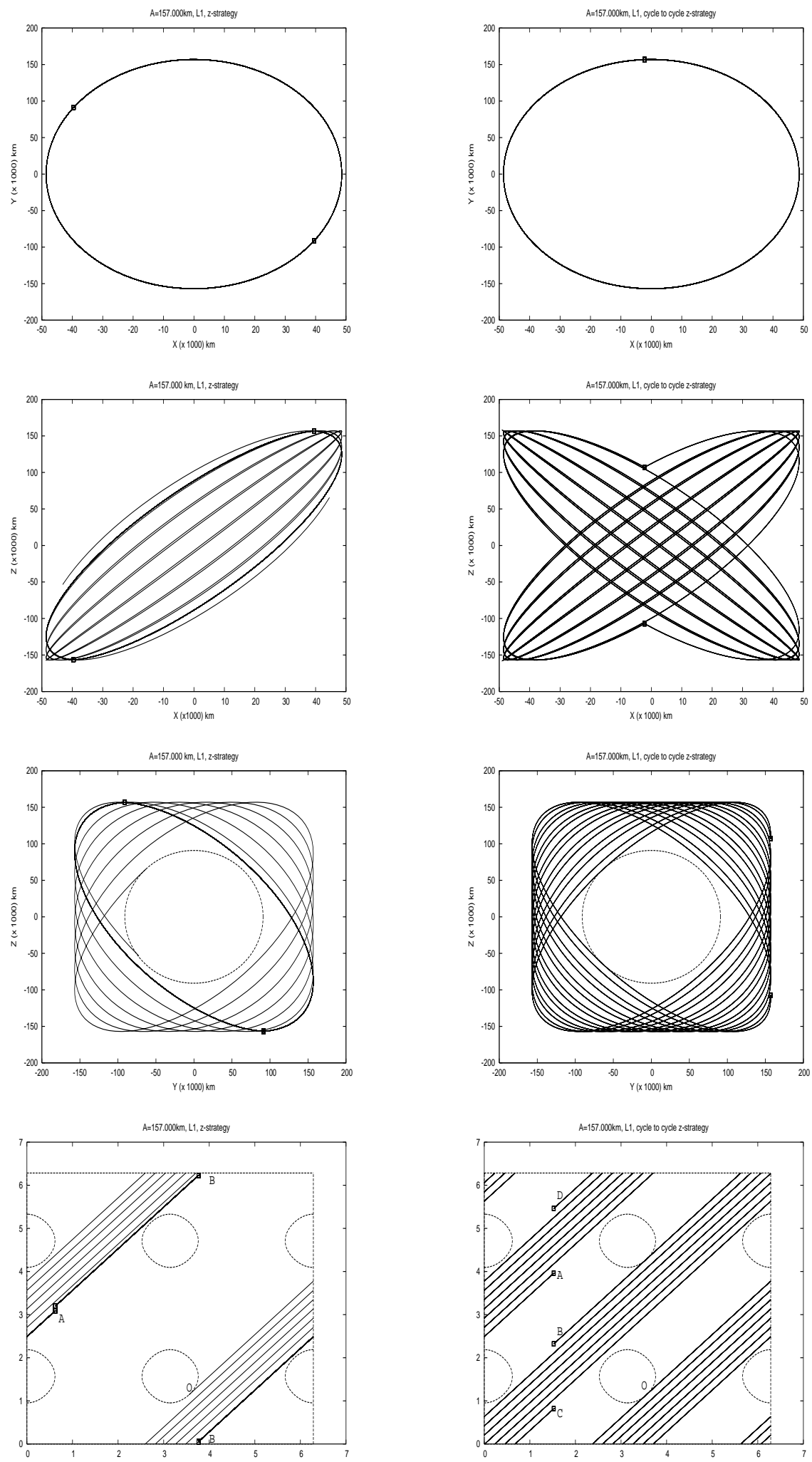

Figure 14: Left. Example of the Pernicka and Howell strategy represented in the EPP for an orbit with $A=157000 \mathrm{~km}$ and $R=90000 \mathrm{~km}$ about $\mathrm{L}_{1}$. Departing from a tangent trajectory at $\mathrm{O}$, the satellite evolves till just before entering the exclusion zone, then it enters in a cycle with two $z$-maneuvers per revolution (four per year). The maneuvers are applied in two different places, $\mathrm{A}$ and $\mathrm{B}$, and the jump in the $\Psi$ direction in each place is one half of the $\Psi$-distance between two consecutive lines in the EPP corresponding to the same natural motion. The final cycle is seen like an ellipse about the exclusion zone. Maneuvers are stopped when the remaining time without eclipse is enough to finish the mission. Right, the corresponding plots using the LOEWE strategy with $z$-maneuvers. Each cycle closes with a maneuver at $\mathrm{A}$ or $\mathrm{C}$ which jumps to B or D respectively. The whole pattern repeats again after two cycles. In both cases the places of the maneuvers are marked with a small box. 
The problem is that these ideal locations for combined maneuvers are usually inside the exclusion zones, or very close to them. Thus, either it is not possible to use them as maneuver phases because this would imply entering the exclusion zone, or if they are outside the prohibited regions, they make the satellite jump to a trajectory which crosses them soon after the change of amplitudes, which does not suit LOEWE philosophy.

Since when the satellite is in a Lissajous orbit each $t$ is associated with a pair of effective phases, we only need to represent the convenient regions in the EPP. Let us summarize the results obtained in the following sections.

\subsubsection{Increasing the size of a square Lissajous with a combined maneuver}

As we saw in (12) and in (19), for this kind of transfers there is no limit, neither for the time at which we perform the maneuver, nor for the size of the final Lissajous. Assuming that the combined maneuver is performed at the point $\left(\Phi_{m}, \Psi_{m}\right)$, we have to say that not for all the points we have an improvement, moreover the improvement depends on the ratio $A^{f} / A^{i}$.
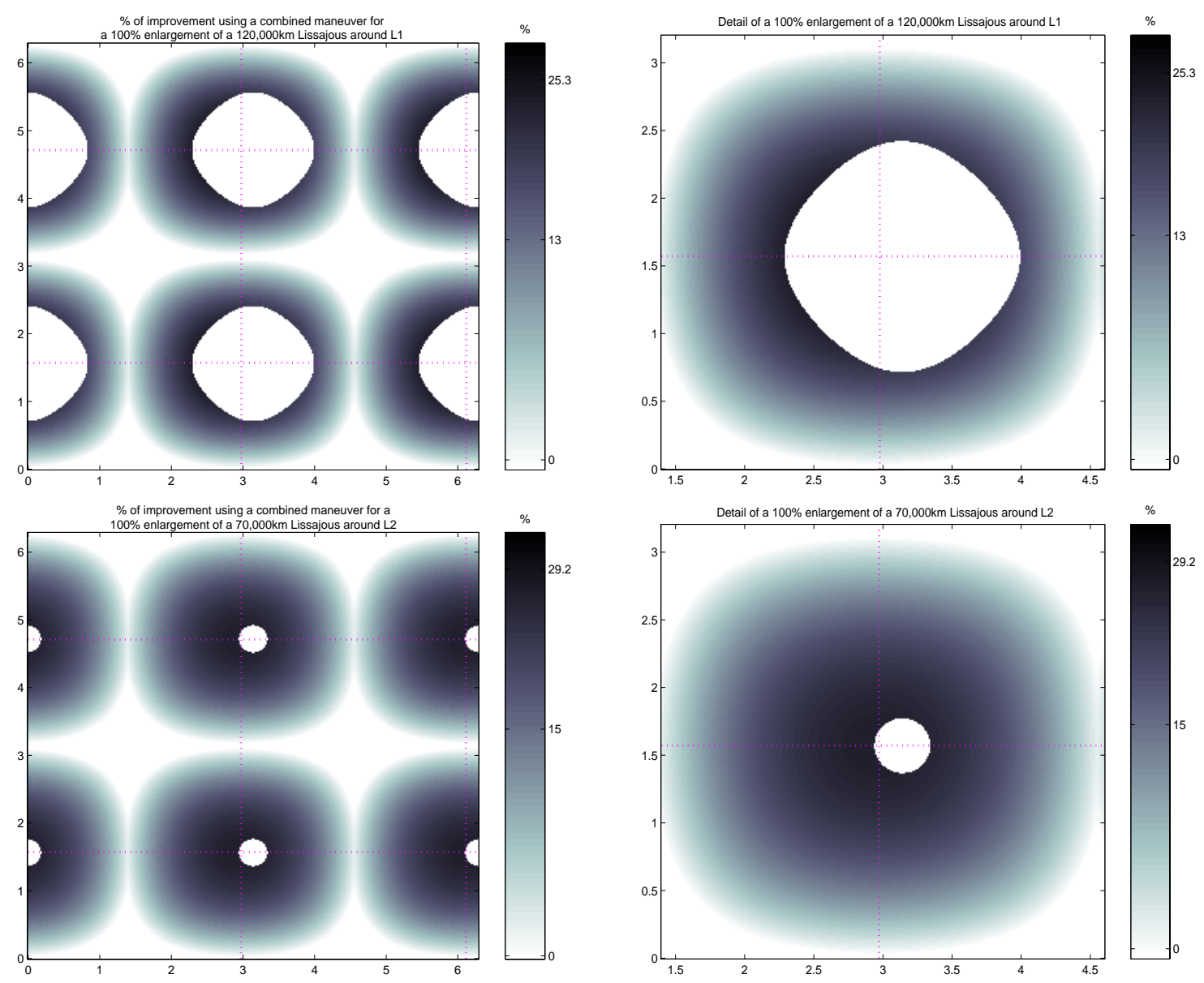

Figure 15: Left. $I(\tau)$ for each point $\left(\Phi_{m}, \Psi_{m}\right)$ when a combined maneuver is used to enlarge a square Lissajous orbit. Right, detail of the left figure about an exclusion zone. Up, case example around $\mathrm{L}_{1}$ from $120000 \mathrm{~km}$ to $240000 \mathrm{~km}$ amplitude. Down, case example around $\mathrm{L}_{2}$ from $70000 \mathrm{~km}$ to $140000 \mathrm{~km}$. Dotted lines in the figures represent the optimal places for the maneuvers to reduce the in-plane or out-of-plane amplitudes.

As an example, figure 15 shows which are the effective phases where executing a combined maneuver leads to an improvement in cost for a $100 \%$ enlargement of a $120000 \mathrm{~km}$ square Lissajous around $\mathrm{L}_{1}$. White zones are either exclusion zones, which we do not consider as valid phases, or phases where it is cheaper to make the transfer using two maneuvers. The behavior is similar for a transfer in the vicinity of $\mathrm{L}_{2}$ as we can see in the 
same figure where we represent a transfer from $70000 \mathrm{~km}$ to $140000 \mathrm{~km}$ square Lissajous of amplitude (again a $100 \%$ enlargement). Note that the improvement essentially depends on the distance to the intersection between the vertical and horizontal dotted lines which correspond to the optimal places for the separate maneuvers. An example of the profile of the improvement function along the optimal coordinate lines is displayed in figure 16.

Other enlargements would have similar pictures, with regions surrounding the exclusion zones as the best improvement rate zones. The maximum $\%$ of improvement is around $30 \%$, which represents a significant reduction in cost.
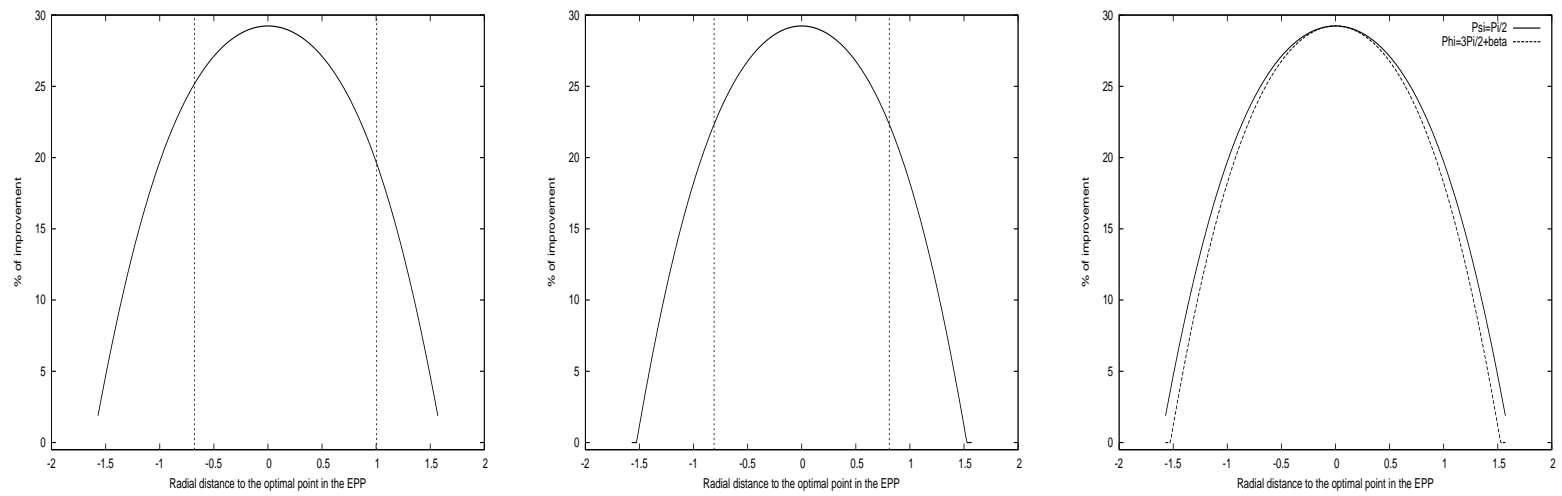

Figure 16: Profiles of the improvement of cost function along the lines of optimal places for in-plane or out-of-plane maneuvers. Left, along the $\Phi$ phase. Middle, along the $\Psi$ phase and right, superposition of both plots. The zero value corresponds to the intersection point of the optimal lines and the vertical dotted lines mark the exclusion zone. The example corresponds to the enlargement maneuver of a square Lissajous around $\mathrm{L}_{1}$ from $120000 \mathrm{~km}$ to $240000 \mathrm{~km}$.

\subsubsection{Decreasing the size of a square Lissajous with a combined maneuver}

The reduction of the amplitude of the Lissajous is subjected to some restrictions. According to (12) and (19) separated transfer maneuvers in the $x y$ and $z$ directions are only possible at some time intervals. That is, for some values of the effective phases. More precisely, for each $A^{f} / A^{i}$ ratio, the effective phases $\left(\Phi_{m}, \Psi_{m}\right)$ for which a combined maneuver is possible are the ones that satisfy,

$$
\left|\cos \left(\Psi_{m}\right)\right|<\frac{A^{f}}{A^{i}}, \quad\left|\cos \left(\Phi_{m}-\beta\right)\right|<\frac{A^{f}}{A^{i}},
$$

which essentially express the idea that, just moving the velocity, one cannot jump from a pendulum oscillation with a certain amplitude to a smaller one when the pendulum is above the required small amplitude.

These pair of equations define two vertical and two horizontal strips, in whose intersection we find the possible effective phases where to perform the transfer maneuver. The more we want to reduce the Lissajous size, the thinner the strips are, and the other way round, as we see in figure 17. In this figure we also observe that the permitted regions always interfere with the exclusion zones. This fact reduces even more the possible places for the maneuver, as we never will use phases inside the exclusion zones as maneuver phases.

The maximum reduction rate (MRR) defined as the maximum value of $\left(A^{i}-A^{f}\right) / A^{i}$ for a given pair of effective phases can also be computed using the equations 27 . In percentage, this value is given as,

$$
\operatorname{MRR}(\Phi, \Psi)=100 \times(1-\max (|\cos (\Psi)|,|\cos (\Phi-\beta)|)),
$$

and it is represented in figure 18 , both around $\mathrm{L}_{1}$ and $\mathrm{L}_{2}$.

As it was observed for enlargement maneuvers, the zones in the EPP which produce a greater improvement in cost are those surrounding the exclusion zones. So again, either upper or lower tangential points seem to be good places to execute a combined maneuver as shown in figure 19. However, as the reduction rate increases, the possible effective phases for a combined maneuver become more restricted. It is even possible that for certain reduction rate and relative amplitude, all (or almost all) the effective phases where a combined maneuver is permitted are inside the exclusion zones. 

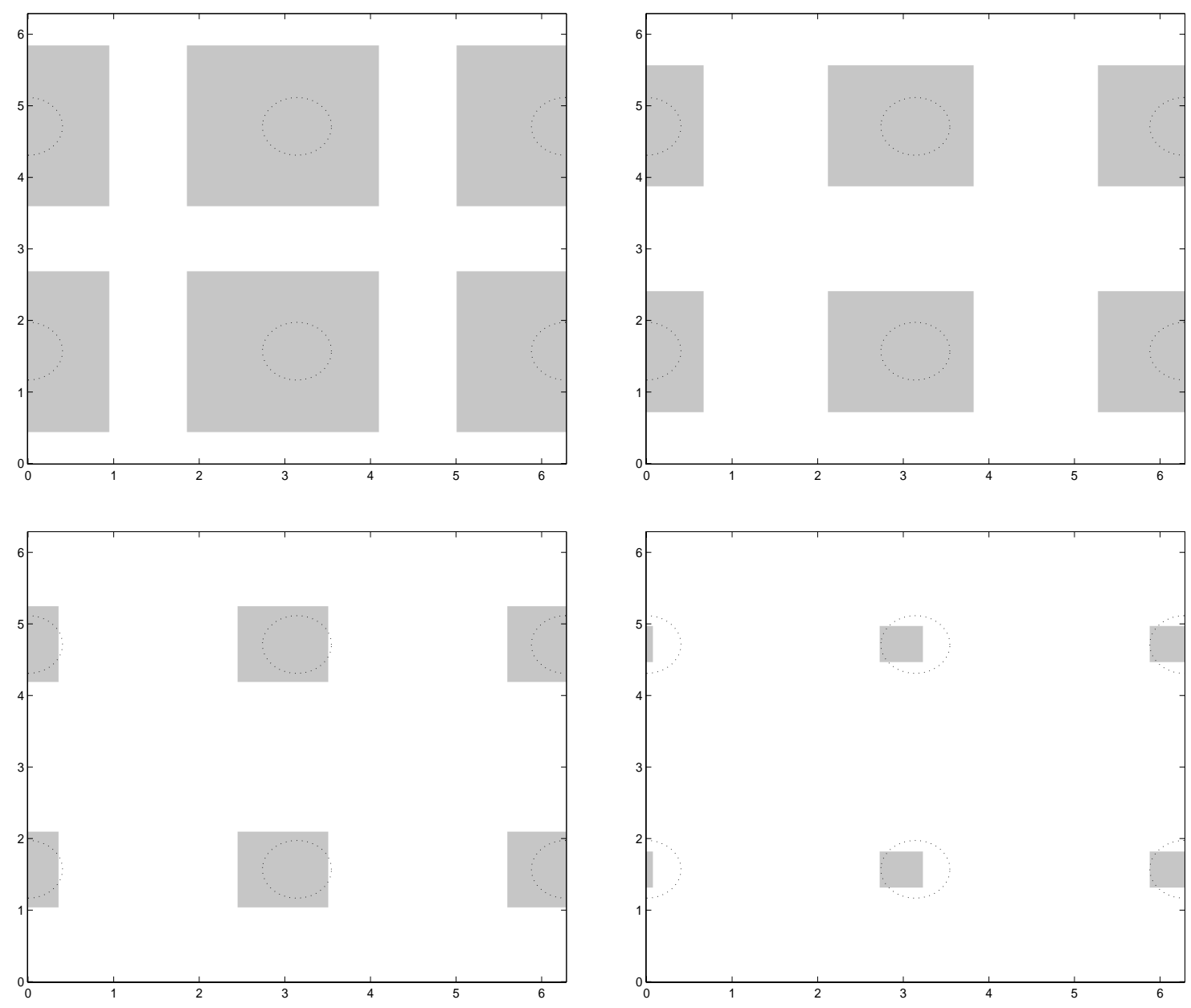

Figure 17: Possible $\left(\Phi_{m}, \Psi_{m}\right)$ where a combined maneuver can give a certain reduction of the Lissajous amplitude. (represented reductions $\left(A^{i}-A^{f}\right) / A^{i}$ of $10 \%, 25 \%, 50 \%$ and $75 \%$ ).
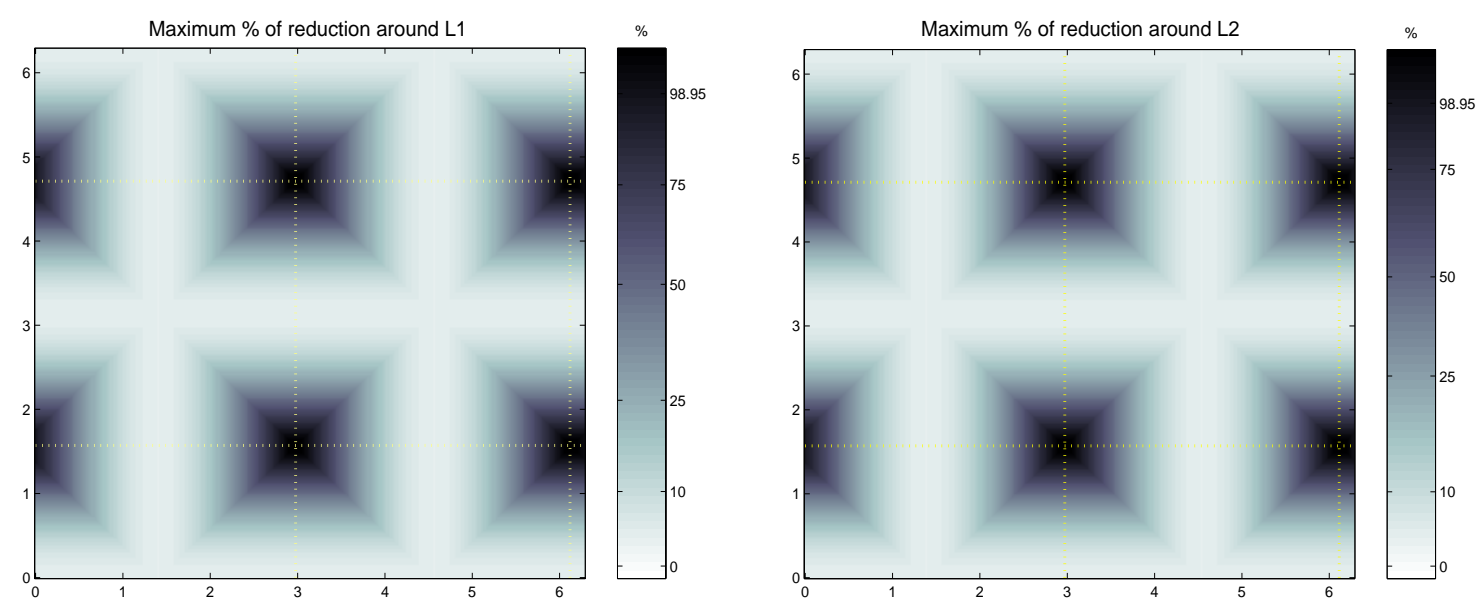

Figure 18: Maximum possible reduction of the amplitude using a combined maneuver, as a function of the effective phases. Right, $\mathrm{L}_{1}$ case. Left, $\mathrm{L}_{2}$ case. 

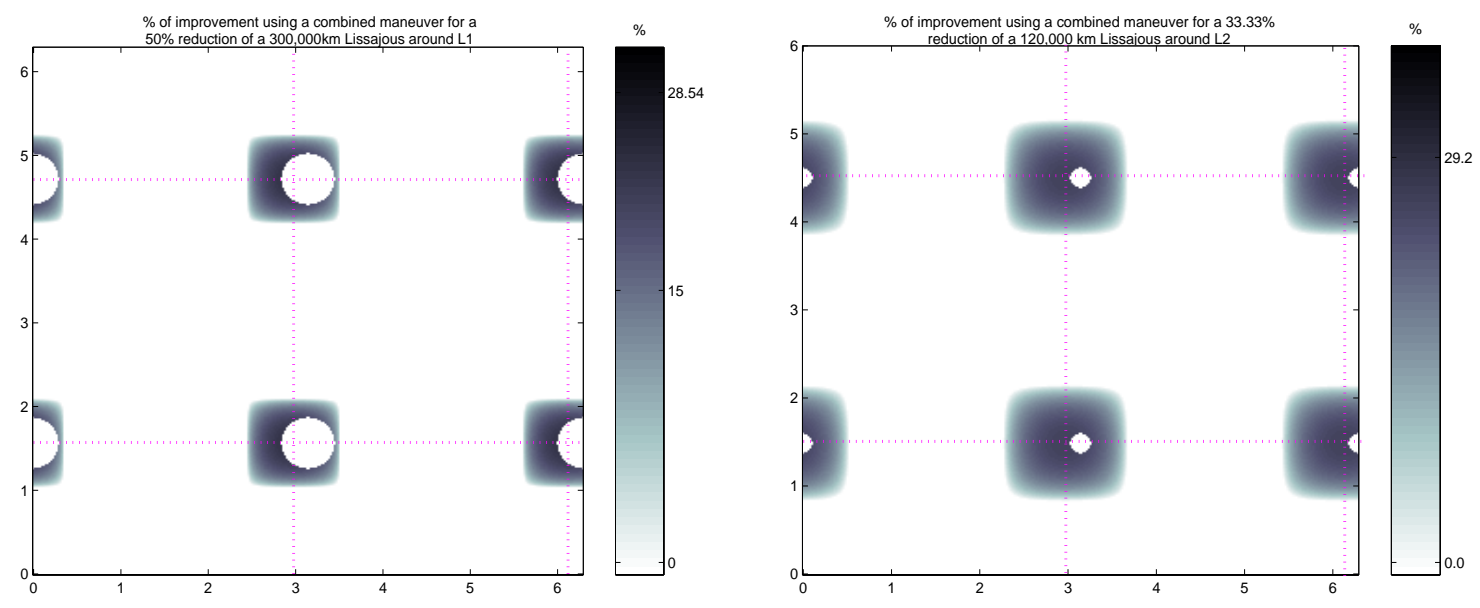

Figure 19: $I(\tau)$ for two examples of reduction of amplitude. Left, from $300000 \mathrm{~km}$ to $150000 \mathrm{~km}$ amplitude square Lissajous about $\mathrm{L}_{1}\left(50 \%\right.$ of reduction). Right, from $120000 \mathrm{~km}$ to $80000 \mathrm{~km}$ amplitude square Lissajous about $\mathrm{L}_{2}(33 \%$ of reduction). The profiles of the function along the optimal coordinate lines look similar to the ones in figure 16 and are not displayed.

\subsubsection{Eclipse avoidance in combined maneuvers to change the amplitude}

Given the initial and final amplitudes, the current objective is to make a transfer using a combined maneuver at $\left(\phi_{m}, \psi_{m}\right)$ in such a way that the satellite remains beyond a certain distance $R$ of the Sun-Earth line (the $x$-axis in the RTBP) during the transfer. For this objective we do not interfere with the maneuvers we have been studying; i.e. we just take advantage of the ones that let us fulfill the two requirements: make a transfer from one amplitude to another and do not violate the exclusion zone.

Given $A^{(i)}$ and $A^{(f)}$, to generalize these results it is necessary to know whether there exist initial phases which: (a) yield a trajectory with no violation of the exclusion zone and (b) give the best possible improvement in cost for a combined maneuver instead of two maneuvers. Again the EPP representation helps us in the design of the mission. Taking as starting point the zones where the improvement is positive using a combined maneuver, it is a matter of just selecting the places where we have maximum time without hitting the exclusion zone once the maneuver is performed. A couple of examples are represented in figure 20. In these figures we represent the time without crossing the $\mathrm{L}_{2}$ exclusion zone $(14000 \mathrm{~km})$ as a function of the effective phase where we perform the maneuver. The darkest zones are the most convenient in terms of time free of eclipse and since the best improvement occurs when the maneuver is executed closer to the intersection of the vertical and horizontal dotted lines, optimal places for the transfer are the closest darkest points to these intersections. In figure 21 we present an example of an enlargement of a square Lissajous about $\mathrm{L}_{2}$.

\section{Conclusions}

Methodologies to make transfers between Lissajous orbits about the collinear libration points using one maneuver have exhaustively studied and, in particular, cheap strategies to avoid the exclusion zone have been obtained. Part of the study has been successfully implemented in the design of the FIRST/Plank mission of the European Space Agency. The effective phases plane has been introduced as a new and promising tool for libration point mission design. Its main advantage is that Lissajous orbits, either linear or nonlinear, appear as straight lines in the EPP representation and so, the bases for nonlinear approaches are the same, only the shape of the exclusion zones appear slightly perturbed. Work on the nonlinear problem to take advantage of this action-angle representation for different purposes is in progress. 

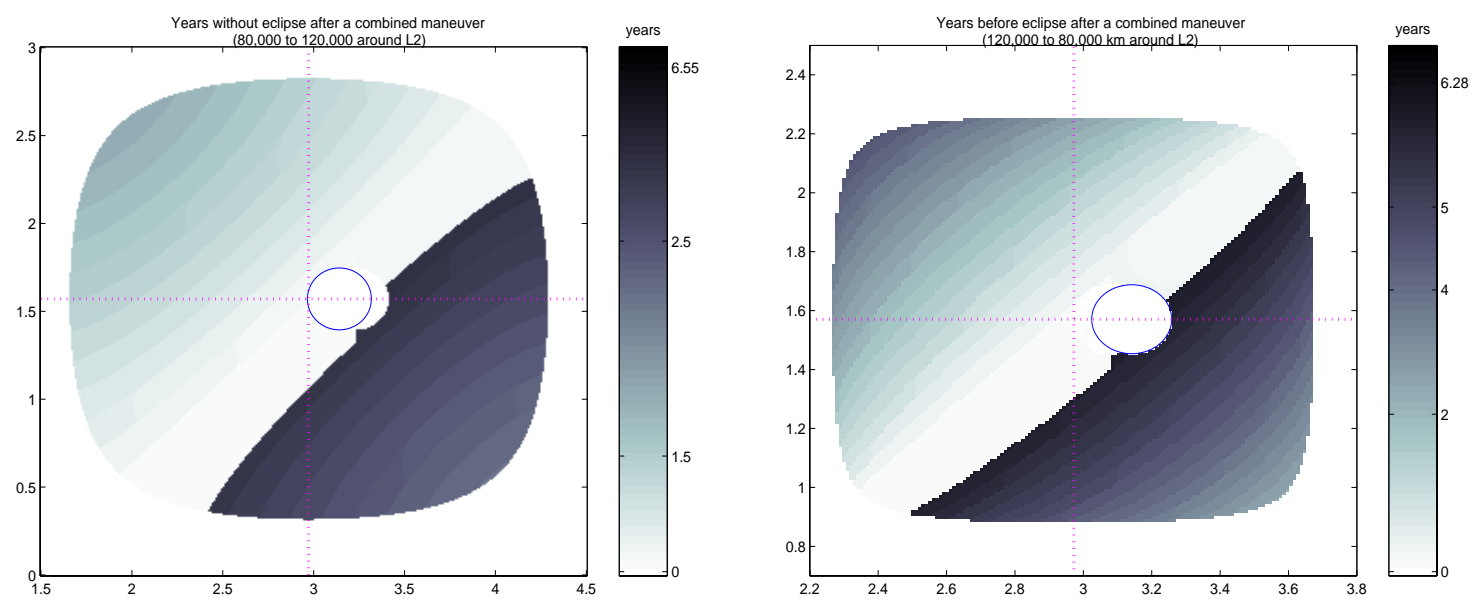

Figure 20: Maximum time interval without crossing the exclusion zone for a combined maneuver to change the amplitude of a square Lissajous. Both examples are details about an exclusion zone in the EPP for the $\mathrm{L}_{2}$ case with $R=14000 \mathrm{~km}$. Left, enlargement of the amplitude from $80000 \mathrm{~km}$ to $120000 \mathrm{~km}$. Right, reduction from $120000 \mathrm{~km}$ to $80000 \mathrm{~km}$. Optimal places for the transfer without crossing the exclusion zone are the darkest places closest to the intersection of the vertical and horizontal dotted lines which represent the optimal places for the separate maneuvers.
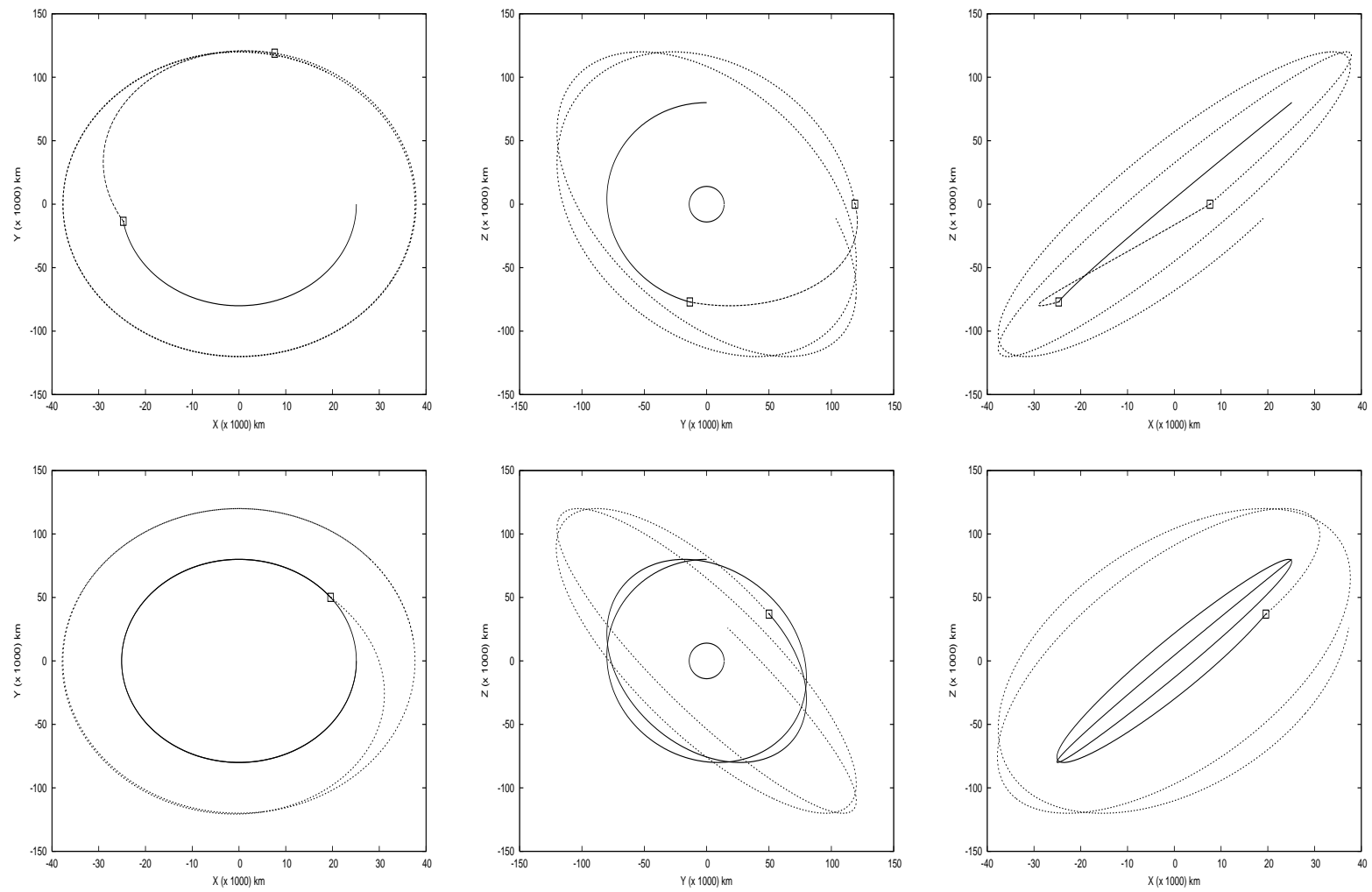

Figure 21: Example of a transfer from a square Lissajous about $\mathrm{L}_{2}$ with $A=80000 \mathrm{~km}$ to $A=120000 \mathrm{~km}$. Top, using two separate maneuvers at the optimal places with total cost $30.33 \mathrm{~m} / \mathrm{s}$. Bottom, using an optimal combined maneuver with cost $23.33 \mathrm{~m} / \mathrm{s}$. A $20.79 \%$ of improvement in the delta-v cost. Maneuvers are marked with a box. 


\section{Acknowledgments}

This research has been supported by the Spanish grant BFM2003-09504, and the Catalan grants CIRIT 2001SGR-

70 and 2003XT-00021. E.C. Acknowledges the fellowship AP2002-1409 of the Spanish Government.

\section{References}

[1] J. Cobos, M. Hechler, FIRST/Planck Mission Analysis: Transfer to Lissajous Orbits Using the Stable Manifold, MAS WP 412, ESOC December 1998.

[2] J. Cobos, J.J. Masdemont, Astrodynamical Applications of Invariant Manifolds Associated with Collinear Libration Orbits. Proceedings of the Conference Libration Point Orbits and Applications, pp. 253-268, World Scientific, 2003.

[3] R.W. Farquhar, Lunar Communications with Libration Point Satellites, Journal of Spacecratf and Rockets, Vol 4, No 10, pp 1383-1384, 1967.

[4] R.W. Farquhar, Comments on 'Optimal Controls for Out-of-Plane Motion about the Translunar Libration Point', Journal of Spacecraft and Rockets, Vol 8, No 7, pp 815-816, 1971.

[5] R.W. Farquhar, D.P. Muhonen, C. Newman, H.Heuberger, The First Libration Point Satellite. Mission Overview and Flight History, AAS/AIAA Astrodynamics Spec. Conf., 1979.

[6] R.W. Farquhar, The Role of Sun-Earth Collinear Libration Points in Future Space Exploration. AAS Annual Meeting, 1999.

[7] G. Gómez, A. Jorba, J.J. Masdemont, C. Simó, Dynamics and Mission Design near Libration Points. Vol 3, Advanced methods for collinerar Points, World Scientific Pub., xvi+187, 2001.

[8] G. Gómez, A. Jorba, J.J. Masdemont, C. Simó, Study of the Transfer between Halo Orbits, Acta Astronautica, Vol 43, No. 9-10, 493-520, 1998.

[9] G. Gómez, J.J. Masdemont, C. Simó, Quasihalo Orbits Associated with Libration Points, The Journal of the Astronautical Sciences, Vol 46, No 2, 135-176, 1998.

[10] T.A. Heppenheimer, Optimal Controls for Out-of-Plane Motion about the Translunar Libration Point, Journal of Spacecraft and Rockets, Vol 7, No 9, 1087-1092, 1971.

[11] A. Jorba, J.J. Masdemont, Dynamics in the Center Manifold of the Collinear Points in the Restricted Three Body Problem, Physica D, 132, 189-213, 1999.

[12] M. Hechler, J. Cobos, FIRST Mission Analysis: Transfers to Small Lissajous Orbits around L $L_{2}$, MAS WP 398, ESOC July 1997.

[13] M. Hechler, J. Cobos, FIRST/Planck and GAIA Mission Analysis: Launch Windows with Eclipse Avoidance Manoeuvers, MAS WP 402, ESOC December 1997.

[14] M. Hechler, J. Cobos, M. Belló-Mora, Orbits Around L for the FIRST, PLANCK, and GAIA Astronomy Missions, presented at 50th IAF Congress, Amsterdam, Oct. 1999.

[15] K.C. Howell, B.T. Barden, R.S Wilson, M.W. Lo, Trajectory design Using a Dynamical Systems Approach with Applications to Genesis, AAS paper 97-709, AAS/AIAA Astrodynamics Specialist Conference, Sun Valley, Idaho.

[16] K.C. Howell, H.G. Pernicka, Numerical Determination of Lissajous Trajectories in the Restricted Three body Problem, Celestial Mechanics, Vol 41, No 1-4, 107-124, 1988.

[17] H.G. Pernicka, K.C. Howell, Sun-Earth Libration Point Trajectories that Avoid the Solar Exclusion Zone, The Journal of the Astronautical Sciences, Vol 38, No 3, 269-288, 1990. 
[18] H. Poincaré, Les Méthodes Nouvelles de la Mécanique Céleste, Gauthier-Villars, 1892. 1893, 1899.

[19] J.D. PorTer, Final Report for Lunar Libration Point Flight Dynamics Study, NASA-GSFC Contract NAS5-11551, General Electric Co. April 1969.

[20] D.L. Richardson, A Note on a Lagrangian Formulation for Motion about the Collinear Points, Celestial Mechanics 22, 231-236, 1980.

[21] The TPF Science Working Group. The Terrestrial Planet Finder: A NASA Origins Program to Search for Habitable Planets. JPL Publication 99-3, 1999.

[22] F. C. Vandenbussche, P. Temporelli, The Trip to the L1 Halo Orbit, ESA Bulletin 88, 1996.

[23] V. Szebehely, Theory of orbits, Academic Press, 1967. 